\title{
Zur Textgeschichte des lateinischen Paulus.
}

\section{Teil: Die direkte Überlieferung.}

Von Ernst Diehl in Innsbruck.

Das fatalistische Urteil des Hieronymus in seinem Geleitbrief zur Evangelienübersetzung an Papst Damasus über die latina exemplaria der hl. Schrift, tot sunt paene quot codices - eine Behauptung, deren wissenschaftliche Berechtigung selbst für Hieronymus' Zeiten zu prüfen wäre (s. S. I00 Anm.) - wirkt wie ein Verhängnis fort bis auf den heutigen Tag: Werden und Entwicklung des lateinischen Bibeltextes blieben trotz aller Mühe und allen Scharfsinns, die dem Problem gewidmet wurden, ungeklärt. Selbst dem geschärften Blick eines geschulten Beobachters bot sich etwa nachstehendes Bild:

Dem unentwirrbaren Chaos von Varianten 'altlateinischer' Bibelübersetzungen, der sogenannten 'Itala' (it), trat um das Jahr 380 in Hieronymus' Vulgata (vg) ein im Grunde einheitlicher und konstanter Text entgegen. Wohltuende Ruhe und erfreuliche Sicherheit machten damals dem unstäten Fluß und steten Schwanken ein ersehntes Ende. Die unleugbaren Beziehungen der vg zur it rechtfertigten sich ja aus Hieronymus' eigenen Worten, 'er habe alte griechische Codices verglichen, sinnstörende Lesungen verbessert, das Übrige, wie es war, unverändert gelassen' (praef. euang.). Daß die vg den völligen Bruch mit der Vergangenheit bedeuten sollte, war jedem Zweifel entrückt.

Damit ist das Problem aber keineswegs gelöst: denn Hieronymus gibt keine bestimmte Auskunft über seine Vorlage, die doch auch eines jener tot exemplaria gewesen sein mul. Wir wissen nicht, auf Grund welchen Rechtstitels ein Exemplar zu solcher Würde gelangte, wenn alle anderen so gar verschieden waren. Und wenn Hieronymus nur sinnstörende Fehler (wie $R m$ I $2{ }_{11} I \operatorname{Tim} 3_{1} \cdot 5_{19}$ ) ausmerzte, wes Alters, Ursprungs und Namens war dann der übrige Text? $\mathrm{Ob}$ und wie die 'vg' aus einer bekannten oder unbekannten 'altlateinischen' Übersetzung oder einer 'Urvulgata' hervorging, blieb trotz der Untersuchungen von Harnack, Wordsworth u. White u. v. a.

Zeitschr. f. d. nentest. Wiss. 20. Band 1921. 
nicht minder ein Rätsel, wie die noch heute offene und strittige Frage nach der Einheit oder Mehrheit selbständiger altlateinischer Bibelübersetzungen ${ }^{1}$ ).

An die Stelle solch unerläßlicher, grundlegender Forschungen traten vielfach umfangreiche Erörterungen über minutiöse $\mathrm{Ab}$ weichungen und Eigentümlichkeiten einzelner it- und einzelner vgCodices, um intimste verwandtschaftliche Beziehungen dieser und jener Hs (z. B. von $D d: E e, G g: F f$ ) zu klären, genaueste Stammbäume zu errichten ${ }^{2}$ ). Untersuchungen über Reihenfolge der Bücher, Abtrennung der Zeilen, Kola und Wörter, über neue Absätze, vorhandene oder fehlende Initialen, über Gebrauch immer wiederkehrender Partikeln, Konjunktionen und Redeteile, Äußerlichkeiten und Zufälligkeiten im großen Zuge der Überlieferung eines vieltausendmal abgeschriebenen und verarbeiteten Textes, können nie und nimmer den Weg zur Urform und zum Archetypus weisen.

Ein zweiter Irrtum. Das it-Problem zu lösen setzte man meist den Hebel an bei den Schriften, deren direkte wie indirekte Überlieferung die reichste Ausheute versprach und tatsächlich brachte: bei den Evangelien . . . und man versank und erstickte im Material. Und doch: gelingt es, auch nur für ein Buch des NT die Entwicklungsformel des Lateiners $\mathrm{zu}$ finden, ist der Bann auch für die übrigen Bücher gebrochen. Denn nichts verlautet über ungleiche Behandlung der einzelnen Bücher etwa des NT anläßlich ihrer Übertragung ins Lateinische.

Darum war es ein richtiger und fruchtbarer Gedanke von Peter Corssen ${ }^{3}$ ) und Friedr. Zimmer ${ }^{4}$ ), an einem Musterbeispiel, dem Galaterbrief, die Textgeschichte des lateinischen NT zu ergrünclen und zu schildern. Die Verwirklichung des Gedankens ermutigte allerdings nicht zu neuen Versuchen. Abgesehen von einem Grund-

1 Wichtigste Literatur bei Eb. Nestle Einführung in das gr. NT ${ }^{3}$ (Göttingen 1909) S. 123 ff. 138 ff., C. R. Gregory Textkritik des NT II 594 ff., M. Schanz Gesch. d. röm. Lit. III ${ }^{2}{ }_{4} 83$ ff., W. S. Teuffel-Kroll Gesch. d. röm. Lit. ' III S. I3I ff. s. auch H. v. Soden Die Schriften des NT I 2007 ff., Ad. Harnack Gesch. d. altchr, Lit. 2, 2 (1904) $296 \mathrm{ff}$. 'Über die lat. Bibel zur Zeit Tertullians und vor Tertullian'. Leider konnte v. Harnacks Abhandlung über die Vulgata des Hebräerbriefes nicht mchr eingesehen werden.

${ }^{2}$ S. v. Soden I $1937 \mathrm{ff}$. Nestle ${ }^{8}$ I 45 ff.

3 Epistula ad Galatas ad fidem optimorum codicum Vulgatae recognouit, prolegomenis instruxit, Vulgatam cum antiquioribus uersionibus comparauit Berol. 1885.

4 Der altlateinische Text des Galaterbriefes als Grundlage für einen textkritiscben Apparat der Vetus Latina. Theolog. Studien u. Skizzen aus Ostpreußen, Königsberg I (1887) I ff. 
irrtum Zimmers, der die Zeugen zum Teil einander unterordnete statt koordinierte, steigern der vor den Augen des Lesers sich entfaltende scheinbare Reichtum und die große Mannigfaltigkeit der Überlieferung Verwirrung und Unsicherheit, statt sie zu beheben: das Abweichende und Trennende ist hier wie allerorten in den Vordergrund gerückt und überschätzt, das Gleiche und Einigende zurückgedrängt und mißachtet.

Nichtsdestoweniger waren Corssen und Zimmer auf der rechten Fährte. Einen Einblick oder gar einen Einbruch in das Labyrinth der Wege und Irrwege, Kreuzungen und Sackgassen, ein Erfassen der Brennpunkte und Ruhepausen des Werdeganges der lateinischen Bibel verheißen lediglich die Paulinischen Briefe. Ihre direkte wie indirekte Überlieferung ist trotz aller Fülle quantitativ zu bewältigen; sie ist aber auch so abwechslungsreich, dab die Textgeschichte des lateinischen 'Apostolos' der Lösung und Entwirrung selbst verwickelterer Gefüge Mittel und Wege weisen muß.

Die direkten Zeugen des altlateinischen Textes der Paulusbriefe sind edierte und nicht edierte Hss; ihnen mögen gleichgestellt werden einzelne oder alle Briefe Vers für Vers zitierende und erläuternde Kommentare sowie systematische, wortgetreue Exzerpte. Die indirekte Überlieferung stützt sich auf die gesamte vorhieronymianische Literatur, auf die Werke des Hieronymus und seiner Zeitgenossen, und auf eine stattliche $\mathrm{Zahl}$ jüngerer Autoren bis ins 8. und 9. Jh., bis zu Beda, dem Iren, und Sedulius, dem Schotten.

Trotz aller Verschiedenheiten und Abweichungen im einzelnen, besonders in Hebräerbrief, gebührt der direkten Überlieferung ob ihrer Stabilität und Festigkeit im Gegensatz zur Unzuverlässigkeit und Wankelmütigkeit der meisten indirekten Zeugen uneingeschränktes Lob: dieser Vorzug ist nicht relativer, sondern yon absoluter Art: die für den Gelehrten unserer Zeit selbstverständliche peinliche Genauigkeit in der Benutzung und Anführung von Quellen lag den Kirchenvätern und -schriftstellern der ersten christlichen Jahrhunderte fern.

Diese Erkenntnis aber ist für die Anlage einer kritischen Ausgabe des lateinischen Paulus von weittragender Bedeutung. Denn sollte sich die ursprüngliche Einheit des làteinischen Paulustextes in allen Überlieferungsquellen bewahrheiten - und sie besteht, abgesehen von den Autoren, die nachweislich aus eigenem die griechische Bibel übertragen, wie Victorinus von Pettau, Zeno von Verona, Maximinus, der Arianer u. a., tatsächlich, selbst in dem so buntfarbigen Hebräerbrief - treten die indirekten Zeugen insgeșamt bei der 
Rekonstruktion des authentischen Textes gegenüber den direkten Quellen durchaus in den Hintergrund. Das sich auf dieser Basis entrollende Bild vom Werden und von den Schicksalen des Lateiners überrascht den Bearbeiter, der sich zuerst vielfach rat- und hilflos einem undurchdringlichen Dickicht und wilden Gewirr von Lesarten gegenüber sah, durch seine Klarheit, Übersichtlichkeit und Einheitlichkeit immer wieder ${ }^{1}$.

\section{Die Quellen der sog. Itala der Paulinischen Briefe: I. Die Codices.}

A. Edierte Texte ${ }^{2}$.

I) $D d=$ Cod. Paris. gr. Io7 saec. VI $=$ Codex Claromontanus ed. C. Tischendorf Lips. 1852. Ersatz für verlorene Blätter bietet die Abschrift $E e=$ Cod. Petersburg. (Muralt 20) saec. IX. Er ersetzt den Lt. I Cor $\mathrm{I}_{4} \mathrm{~g}_{\mathrm{I}} \mid$ det tuba $-{ }_{18}$ gratias ago deo. Einer 2. Tochterhs. von $D d$ nach dessen Korrelstur durch $D^{3}$ entstammen die von Viktor Schultze (Unbekannte Fragmente einer griechisch-lateinischen Bibelhs. München 1904) im Stadtarchiv von Mengeringhausen (Waldeck) entdeckten Bruchstücke $W_{\varepsilon}$ des Epheserbriefes (It. $\mathrm{I}_{5 \rightarrow 13}{ }^{2}{ }_{8-11}$ ) aus einer Hs. des II. Jh., die im 17. Jh. als Umschlag einer Schützenordnung verwendet wurde.

2) $G g=$ Codex Boernerianus Dresden A 445 b saec. IX. Lichtdruck mit Einleitung hrsg. von Alexander Reichart, Leipzig 1909. Geschrieben in St. Gallen von Bischof Marcus bzw. dessen Neffen Moengal (s. Vorbericht S. 14) oder Sedulius Scotus, s. L. Traube O Roma nobilis (Abh. d. bayer. Akad. d. W. philos.-philol. Kl. 19, 1892) 2, 348; dagegen S. Hellmann Sedulius Scotus (Traube's Quellen u. Unters. I, 1906, 148 ${ }_{6}$ ).

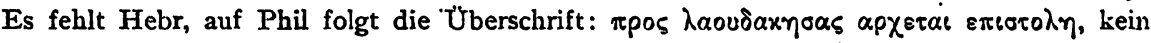
Text. Abschrift oder Schwesterhs. von $G g$ ist $F f=$ Cod. Augiensis saec. IX, jetzt in Cambridge Trinity College $B \mathrm{r} 7,1$. Der lateinische Text ist durchweg der vg angeglichen.

3) $r\left(r_{2}\right):$ a) Cod. Monac. 6436 (Cim. 13 a) saec. V/VI [außer fol. 16, saec. VII $\left(=r_{2}\right)$, das als vollwertiger Ersatz für das verlorene Blatt zu gelten hat $]=$ Fragmenta Frisingensia-M onacensia: die sog. Freisinger Itala hrsg. von Leo Ziegler, Italafragmente der Paulinischen Briefe Marburg 1876 . b) Universit. Bibl. Monac. 4 Cod. Ms. 928 s. Ed. Wölfflin Neue Bruchstücke der Freisinger Itala Münch. Sitz.-Ber. 1893, 2

${ }^{1}$ Ein Beispiel. Die Varianten zu $\mathrm{Rm} \mathrm{II}_{\mathrm{s}}$ (o altitudo diutitiamum usw.), allerorten und ungezäblte Male zitiert, würden heute bei Sabatier eine Großfolio-Seitc und mehr füllen. Nach Ausmerzung alles Individuellen, Willkürlichen, Zuffilligen und Fehlerhaften schrumpft die gesamte direkte und indirekte "Textgeschichte" unter Zugnundelegung von $d$ auf die wenigen Bemerkungen zusammen: $e t^{1} \mathrm{dg} \mathrm{m}$ Iren. Cypr. Tert. . . + $\mathrm{n}$

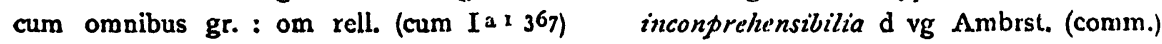
$\ldots+\mathrm{n}$ : inscrutabilia $\mathrm{g}$ gue $\mathrm{m}$ Ambrst. (lemm.) Iren. Cypr. . . $+\mathrm{n}$ indicia $\mathrm{d} g$ Iren. Tert. . . + n cum omnibus gr. : sunt indicia guc vg $\mathrm{m}$ Ambrst. Cypr. $\ldots+n$ allenfalls noch: altitudo d g vg Ambrst. Iren. Cypr. . . + $\mathrm{n}:$ profundum Tert. Hil. Hier. Alle anderen Lesungen sind gewissermaßen ohne Gewäbr und sind nur als Raritaten und, um der Vollständigkeit willen, gesondert zu buchen. Wer jede Variante als 'Tradition bewertete, dem freilich wuchs die Zahl des cxicmplaria ins Unbegrenzte.

${ }^{2}$ Siehe besonders Nestle ${ }^{3} 73$ ff. 134, wo die Literatur verzeichnet ist; vgl. Gregory II 611 ff. 633. 638, v. Soden I 1937 ff. 2012 , H. Lietzmann Hdb. z. NT III I ${ }^{2}$ S. 8 ff. 
S. 253-280: Gal $3{ }_{5}-4 s_{s}=$ fol. 5 I cod. $r 6_{5}-$ Eph $I_{18}=$ fol. $54 \operatorname{cod} r_{0}^{1}$ c) G. Morin Revue Bénédictine 28 (I9II) p. 22 I ss Un nouveau feuillet de l'Itala de Freising (Ms $r$ des Epîtres Paulines), losgelöst aus Cod. Monac. Clem. 28 135. S.' Wiener Sitz. Ber. 169 (IgI2) Abb. V. S. IIO: II Cor $5_{1}-5_{12} \cdot 5_{14}-6_{2} \cdot 3_{3}=$ fol. 40 cod. $r_{0}^{2}$ d) Nichtediertes Bruchstück, losgelöst aus Cod. Monac. lat. 6317 : I Cor $7 ;-12 \cdot 19-26=$ fol. 25 cod. $r$ $13_{13}-14_{5} \cdot 14_{11-18}=$ fol. 32 cod. $r$.

4) $r_{s}=$ Fragmenta Gotuicensia saec. VI/VII auf 2 Pergamentblättern, die als Einbanddeckel eines Codex der Notae Tironianae dienten. Hrsg. von Hermann Roensch Italafragmente des Römer- und Galaterbriefes aus der Abtei Göttweig Z. f. w. Th. 22 (1879) S. 224 ff : Rm $5_{16}-6_{4} \cdot 6_{6-19}$. Gal $4_{6}-19^{\circ}{ }_{22}-5_{2}$.

5) gue = Fragmenta Guelf erbytana saec. VI. s. Franc. Anton. Knittel UIphilae uersionem Gothicam nonnullorum capitum epistolae Pauli ad Romanos ed. Brunsuic. 1762 p. 5 ss, C. Tischendorf Anecdota sacra et profana 1855 p. 153 ss und W. Streitberg Die gotische Bibel I 239-249: Rm II ${ }_{33}-12_{5}$. I $_{21}-13_{5}$. i $_{9}{ }_{9}-15_{13}$.

\section{B. Nichtedierte Texte.}

6) $x_{2}=$ Cod. Oxxon. Bodlei. Laud. ro $8 \mathrm{E} 67$ saec. IX in.: I II Thess. stehen vor Col. (s. Aug. doctr. christ. 2, I3). Der Text bricht ab Hebr. II ${ }_{34}$ effigerıınt aciem gladii.

7) Cod. Ambros. E 26 inf.

8) Cod. Cantabr. Trin. Coll. B ro, 5. saec. IX: I Cor $7_{32}-$ I Thess.

9) Cod: Harleianus I772 saec. IX: Kollationen bei Griesbach Symbolae criticae (Halle 1785) I $326 \mathrm{ff}$ (s. Gregory II 633) : wertlos.

\section{Kommentare.}

I) Marius Victorinus zu. Gal, Eph, Phil. Migne lat. 8. 2) Der sog. Ambrosiaster zu allen Briefen, außer Hebr. Migne lat. I7. 3) Hieronymus zu Phm,

1 Eine Überprüfung der bisher edierten Bruchstücke von $r\left(r_{2}\right)$ ergab neue Lesungen, die in der indirekten Überlieferung eine überraschende Bestätigung fanden: I Cor 1415 fordert der Raum [orabo alıtem et mente] (Aug. gen. ad litt. 12, 9) und psai[lam autem et mente]. 1416 [quando quide]m (Aug.) oder [quoniam quide]m (g Hier.). II Cor $5_{5}$ operatus est (Aug.). $\quad 5_{8}$ inmanentes corpori. $\quad 5_{9}$. [am]bimus (Aug). statt contendimus. $\quad 512$ [nos] $\mid m[$ [et]ipsos [commendamus] statt [con] $\mid m[$ endamus]. $5_{16}$ nonce[ri]mus. $\quad 6_{1}$ suscipiatis (Aug.). $\quad 6_{2}$ [t]emipor[e] ac[ceptabi]li und diem. $7_{11}$ re ip $[s] a$, nicht re $u[e r] a$.

2 Von cod. $r$, der ursprünglich mindestens 12 (nicht II, wie Ziegler annahm) Quat. und I Unio (vielleicht Binio, dann genau 100 fol.) umfaßte, sind 27 fol. teils unversehrt, teils verstümmelt zum Vorschein gekommen, die sich auf die einzelnen Quat. also ver-

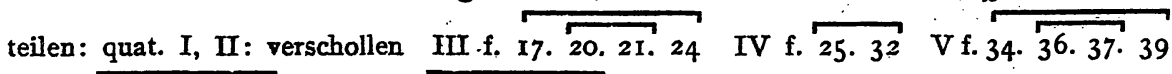
局

VI f. 4 2. 41.45 .47 VII f. 50. 51. 54. 55 VIII f. 60.64 (nicht 59.63) IX bis XIII $f$ 65-100? IX entweder $f .7 I$ oder IX verschollen $X$ entweder $f .74$ oder f. $74 . \overline{75.78} .79$ XI entweder verschollen oder f. 88 XII entweder f. $\overline{90 . \overline{91.94 .} 95}$ oder f. $9 \mathrm{I}$ XIII verschollen. Unsicher wie die Anordnung der erhaltenen Blätter der letzten Quaternionen ist hier auch die Reihenfolge der Briefe: Auf Rm I II Cor Gal Eph Phil I II Thess folgten entweder I II Tim Col Tit Phm [bzw. Tit Col Phm, wie im Canon des Claromontanus] Hebr, oder Hebr (so Aleph) Col I II Tim Tit Phm Zieglers Verteilung (Col I II Tim Tit $\mathrm{Phm}$ Hebr) ist unmöglich. 
Gal, Eph und Titus. 4) Augustinus zu Rm, Gal. 5) Pelagius und Pelagius auctus seu interpolatus $(=$ Ps. Pelagius $=$ Ps. Hieronymus) $\mathrm{zu}$ allen Briefen außer Hebr. 6) Ps. Hieronymus $=$ Ps. Primasius zu Hebr s. E. Riggenbach Histor. Studien zum Hebräerbrief I Leipzig 1907 S. II. 7) Theodori episcopi Mopsuesteni comment. in epist. minores Pauli apost. latine uersus saec. VI. ed. Swete (Cambridge) $1880 / 2$. 8) Be das bzw. Ps. Bedas Bibelkommentare. 9) Sedulius Scotus' Collectaneum in epistolas Pauli (Migne I03, Iff) s. S. Hellmann Sedulius Scotus $147 \mathrm{ff}$ Igo ff. Von den Kommentaren sind eigenen Rechtes der des Marius Victorinus und des sog. Ambrosiaster, die Erläuterungen des Hier. und Aug. sind von den übrigen Schriften dieser Kirchenväter unzertrennlich. Beda (Ps. Beda) und Sedulius Scotus sind unselbständige Zeugen und bald von Aug., bald von Hier., Pelagius u. a. m. (s. Hellmann a. a. O.

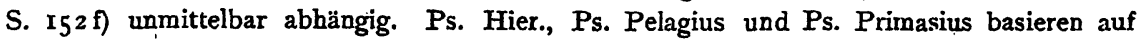
der vg. Die Übersetzung des Rommentars des Theodorus von Mopsuestia nimmt auf keinen lat. Paulus Rücksicht oder stüzt sich auf die $d$ - und $v g$-Formen (Swete s. XII ff). Der von Alex. Souter im Cod. Augiensis CXIX saec. IX in Karlsruhe wiederentdeckte nicht interpolierte Kommentar des Pelagius lehnt sich nach Souter ${ }^{1}$ und Giov. Mercati ${ }^{2}$ an einen der Sixto-Clementina nächstverwandten Bibeltext an.

\section{Excerpte.}

I) Cyprians Testimonia: sie sind von Cyprians anderen Schriften unzertrennlich. 2) Speculum Augustini: Augustins Speculum ist die einzige Schrift Augustins, welche lediglich die Vulgata zitiert, schaltet also für die Geschichte der 'Itala' völlig aus. 3) $m=$ Fragmenta Novi Testamenti ex Libro de diuinis scripturis siue speculum quod fertur s. Augustini. Der Text des Cod. Sessorianus ist hrsg. von J. Belsheim Skrifter udgivne af Videnskabsselskabet in Christiania Hist. filos. Kilasse $1899 \mathrm{Nr}$. 2. Die Sonderausgabe von $S$ ist mit Rücksicht auf $F$ verfehlt. Vgl. Weihrich Corp. Vindob. 12.

IV. Väter- und Schriftstellerzitate.

Die Textmasse der 'altlateinischen' Paulusbriefe stützt sich also einerseits auf $d g$ durchwegs (außer Hebr), gleichzeitig vielfach auf $r\left(r_{2}\right)$ unter stellenweiser Assistenz von $r_{3}$ und gue; andererseits auf Marius Victorinus, Ambrosiaster und m. Dieses Material ist trotz der sachlich und methodisch gerechtfertigten Ausschaltung gewisser Quellen noch so gewaltig, daß sich Kern und Entwicklung der direkten Überlieferung herausschälen lassen müssen, wenn sie überhaupt noch lösbar und faßbar sind. Wird außer diesen Zeugen der Text z. B. des Romerbriefes als Ganzes in die Untersuchung einbezogen, so ist jede im Materialmangel begründete Überraschung ausgeschlossen.

Das Problem der direkten Überlieferung der Paulinischen

1 The Commentary of Pelagius on the Epistles of Paul London 1906 (Proc. of the British Acad. Vol. II) p. $17 \mathrm{f}$.

$=$ Giov. Mercati and A. Souter Journal of theological studies 8 (1907) p. $526 \mathrm{ff}$ 535 f. Vgl. G. Morin Jean Diacre et le Pseudo-Jéróme sur les epîtres de s. Paul Revue Bénédictine $27(1910)$ p. II6. 
Briefe, welches auch vor der vg nicht Halt machen darf, gipfelt in der Beantwortung der Frage:

Ist der lateinische Paulustext einheitlich oder nicht?

Eine Mehrheit selbstherrlicher Texte kann ihren Grund haben entweder in selbständigen, nach Zeit und Ort voneinander unabhängigen Übertragungen aus dem Griechischen - dann müBten sich aber in Anbetracht des reichen griechischen Quellenmaterials auch verschiedene Fassungen des griechischen Apostolos bei den Lateinern trotz aller Überarbeitungen nachweisen lassen - oder in rerschiedenen Rezensionen einer verschollenen Urübersetzung; die auf lateinischem Boden ein Eigenleben geführt haben und jetzt in unseren Quellen fortleben, ohne dab es gelänge, den Archetypus zu rekonstruieren.

Einheitlicher Charakter des lateinischen Paulustextes löst die Frage aus, ob wir den Urtext besitzen bzw. wiederherstellen konnen oder nicht.

Wer einer Mehrheit oder gar Vielheit voneinander unabhängiger Übersetzungen das Wort redet, schuldet eine befriedigende Erklärung für die große Masse der allen Hss gemeinsamen Lesungen ganzer Verse ${ }^{1}$ und Versteile, für die Tatsache also, daß zwei oder mehrere Übersetzer einer und derselben, geschweige mehrerer heterogener griechischer Vorlagen auf einen so einheitlichen Text verfallen konnten. Wohin völlig oder auch nur teilweise selbständige Übertragungen führten und führen mußten, mag eine Gegenüberstellung der Lesungen von Phil $26 \mathrm{ff}$ nach $d, v g$, Cyprian, Tertullian, Marius Victorinus lehren, die alle im wesentlichen auf derselben griechischen Vorlage fußten: s. nächste Seite.

Doch über die allenthalben gleichlautenden Verse und zusammenhängenden Versstücke hinaus erhält das allen Texten gemeinsame Gut einen mächtigen und sicheren Zuwachs durch Ausschaltung gleichwertiger und belangloser synonymer Partikeln, Konjunktionen usw. aus der Zahl der 'Varianten', sofern die Abweichungen durch keine griechische Quelle gestützt sind.

Daß dies Kriterium, mit Umsicht und Vorsicht gehandhabt, Ablehnung selbst synonymer Nomina und Verba, sogar gewisser Konstruktionen als 'Varianten' im Dienste der eigentlichen Textgeschichte rechtfertigen kann, hat eine Analyse der direkten wie indirekten Überlieferung in zablreichen Fällen ergeben: Marius

1 Z. B. Rm I 2-6. 10. x4. 17. 19. 22 f 2 gl 12 f $17-25.32 f$ 12-18. 21. 23. 27. 4 1. 7. 13. $x 5$. 25. 5 I. 5. 5f. 10. 6 2. 5. 10. 181.7 5. 7. x7f. $21-25$ u. s. f. 


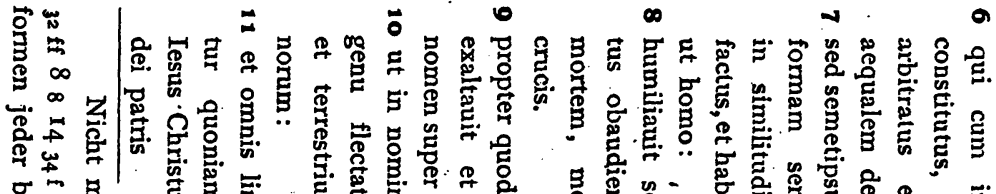

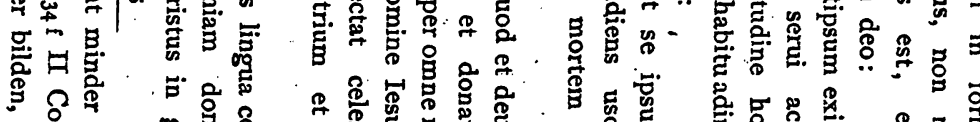

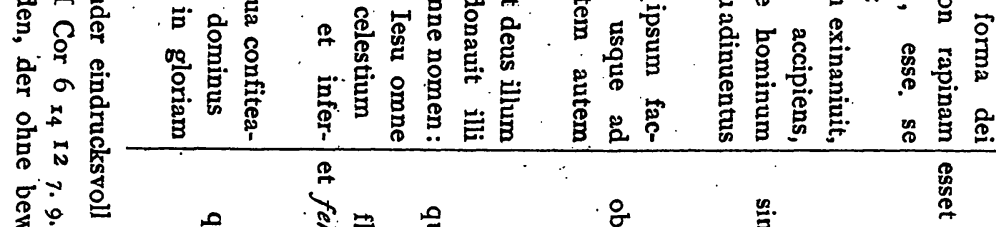

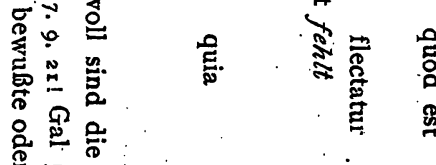

要边

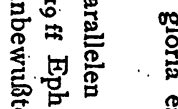

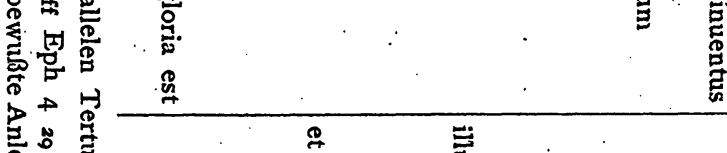

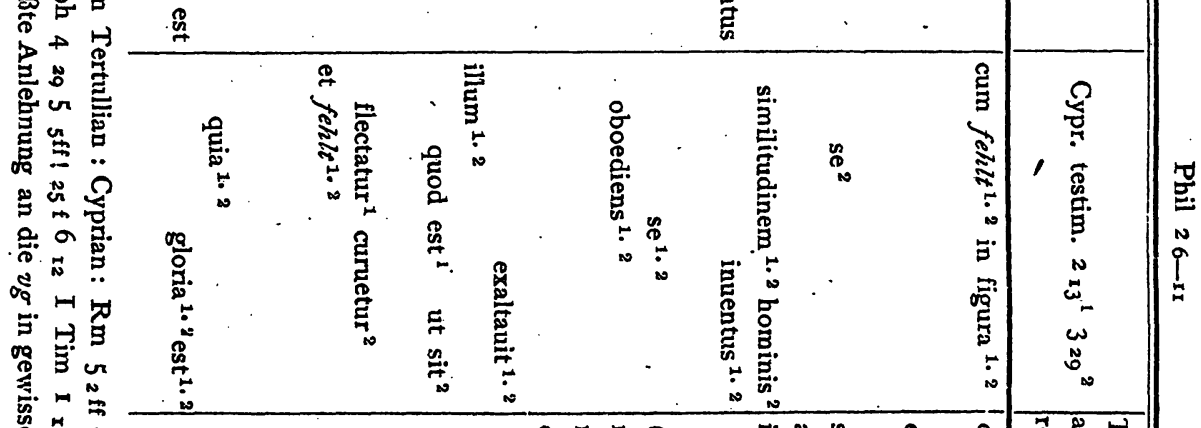

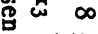

N

宸

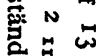

$\stackrel{g}{g}=$

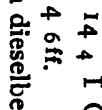

舫

윽

曐:

冡

象

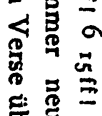

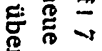

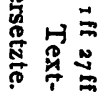

$\stackrel{\infty}{2}$

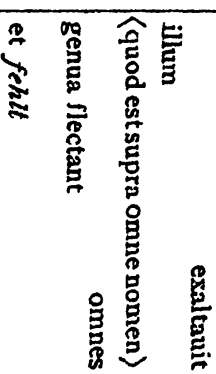

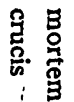

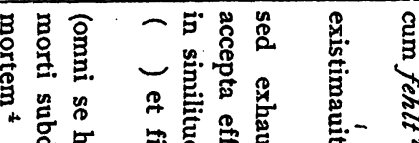

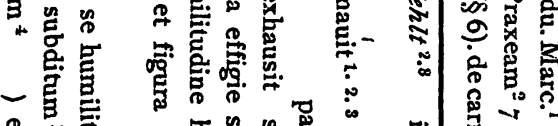

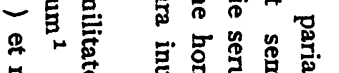

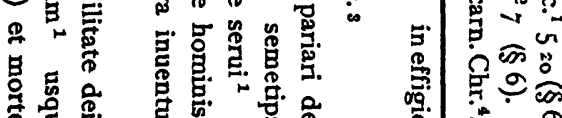

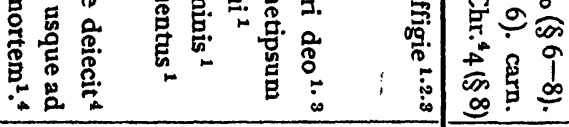


Victorinus z. B. bevorzugt aus eigenem mysterium statt saicramentum, uniuersa st. omnia, sicuti st. sicut, tantummodo st. tantum, er sagt gratulatio, gratulari st. gaudium, gaudere, er ist (de

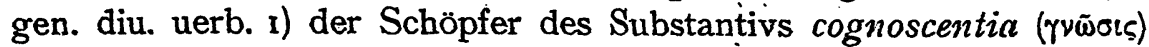
statt scientia ( $\left.\mathrm{Rm}_{\mathrm{II}}{ }_{33}\right)$. Der A m brosia ster lehnt grundsätzlich tribulatio, sanctificatio, sermo, manducare, sustinere $u$. a: ab, die er durch pressura, sanctitas (sanctimonia), uerbum, edere, tolerave ersetzt. Der Frisingensis $(r)$ ersetzt im Hebräerbrief hostia, olocautomata, sempiternus durch sacrificium, holocansta, perpetuus, er will allenthalben selbst von quod und ita nichts wissen. A r d ere Texte ziehen dilectio st. caritas vor, verabscheuen ihren Vorlagen zum Trotz die heidnischen Wörter caerimonia und mysterium. Die unbedingte Abhängigkeit des $g$-Textes vom $d$-Typus wird dadurch nicht in Frage gestellt, daB in den 'Interlinearübersetzungen von $g$ die griechischen Partizipialkonstruktionen viel häufiger als irgendwo anders beibehalten sind oder doch als Variante (k) beigefügt werden.

Die geringe Gewähr derartiger persönlicher Noten und Eigenwilligkeiten gewisser Zeugen für deren Glaubwürdigkeit erhellt weniger aus ibrer Isolierung als aus der Tatsache, daß versehentlich immer wieder an anderen gleichartigen Stellen die Fassung der Mehrheit nicht geändert wurde: Zeugen, die mit solchen stilistischen Eigentümlichkeiten und Schwächen belastet sind, büßen an Vertrauen und Glaubwürdigkeit ein, wenn auch auf völlige Ablehnung nicht erkannt werden dürfte.

Weiterhin ist Mißtrauen gegenüber Textverschiebungen einzelner Quellen geboten, die in keiner griechischen $\mathrm{Hs}$ oder HsKlasse begründet sind. Denn daß Rinnsale verborgener griechischer Quellen in lateinische Texte durchgesickert sein sollten, ist bei unserer heutigen Kenntnis des griechischen NT kaum anzunehmen.

Wie dem auch sei, alle 'Varianten' obgenannter Art können die Selbständigkeit eines lateinischen Textes, dessen unmittelbares Werden aus einer bekannten oder gar unbekannten griechischen Vorlage nicht beweisen.

Wenn aber die spezifische Eigenart einer griechischen Hs oder Hss-Gruppe allen lateinischen Codd. der direkten und der gesamten indirekten Überlieferung aufgeprägt ist, dann ist die grundsätzliche Einheit der griechischen Vorlage bewiesen. Die ungemein nahe Verwandtschaft des Textes $D G(E F)$, der Gruppe $I^{2 x}$ 1026 ff bei Soden, mit dem lateinischen Paulus ist längst erkannt. Es sind lediglich offene Fragen, ob und in welchem Umfang neben $D G$ 
noch andere Hss-Typen zu berücksichtigen sind, ob gar ein Vertreter dieser Gruppe als Urtext zu begrüßen ist.

Die anerkannte Einheit der griechischen Vorlage ließe auch dann die Einheit des lateinischen Paulus vermuten, wenn es kein Codex Graecolatinus wäre, dessen griechische Textgestalt für die lateinische Urform maßgebend wurde. Denn unter diesen Umständen müßte man bei Annahme mehrerer oder gar vieler selbständiger Übersetzér einem jeden dasselbe griechische Original in die Hand zaubern. Wer mit diesem Gedanken spielt, schiebt das ganze Problem aufs tote Geleise, anstatt nach seiner Lösung zu suchen. Trotzdem schließt. das Fortleben eines bestimmten griechischen HsTypus in allen lateinischen Codd. keineswegs aus, daß einer einheitlichen lateinischen Fassung nachträglich Lesarten, selbst ganze Kollationen griechischer Hss. aufgepfropft wurden.

Die Masse des Gleichen, das alle lateinischen Hss. der Paulusbriefe auf Grund der Eigenart der $D$-Gruppe eint, vorzulegen, erübrigt sich, weil individuelle Züge eines noch heute unter uns weilenden Interpreten, die nach menschlichem Ermessen nur einmal geprägt wurden, allen lat. Codd., Commentarii, Exzerpten anhaften. Auf Grund dieser Eigentümlichkeiten des $d$-Textes schließt sich der Ring des Beweises nicht nur für die theoretische Einheit des lateinischen Textes, sondern auch für das tatsächliche Vorhandensein des Urtypus.

Es wäre also unter Beweis zu stellen, daß $g, r\left(r_{2}\right), r_{3}, g u e$, $\mathrm{dab}$ Victorin., Ambrst. und $m$ in ihrer Gesamtheit und jeder einzelne Textzeuge für sich vom $d$-Typus abhängen, mögen sie im Laufe der Zeit noch so sehr mit fremdartigen Elementen durchsetzt worden sein. Denn Fugen und Narben müssen sich zeigen, wenn die gesamte direkte Überlieferung der lateinischen Paulusbriefe als mehrfach revidierter, verbesserter oder verderbter $d$-Typus zu gelten hat. Der Nachweis für das Vorhandensein der beiden polaren Möglichkeiten: $d$ als Archeget aller Zeugen, $d$ als Vorbild jedes einzelnen, erklärt und rechtfertigt auch den allenthalben $z u$ beobachtenden gruppenweisen Anschlu $\beta$ an den $d$-Typus, der im einzelnen nicht belegt $z \mathrm{u}$ werden brauclit. Ich sage $d$-Typus, nicht $d$ : denn ohne das Werden von $d$ selbst in allen Phasen verfolgen zu wollen, $d$ und $D$ sind trotz aller Gleichheiten und Ähnlichkeiten nicht kongruent: jeder von beiden hat, freilich nicht $d$ von $D$ getrennt, ein Eigenleben geführt, $d$ kein kurzes, wenn auch eintoniges: ehe $d$ wurde, loste sich die Urform von $g$ von der minder verderbten Vorlage von $d$ los, die auch für Lucifer von Cagliari u. a. maß- 
gebend ward. Und doch, an Urwüchsigkeit sucht $d$ auch in seiner heutigen Gestalt seines Gleichen. Und wie uns in $D$ etwa das griechische NT des Bischofs Irenaeus von Lyon ( $\dagger$ um 200) vor Augen steht, so führt uns die Analyse der direkten und indirekten Überlieferung des lateinischen Paulus herab bis zum 'Apostolos' des Irenaeus und seiner lateinischen Bearbeitung.

Die geschlossene Abhängigkeit aller Zeugen vom $d$-Typus erhellt aus dem unverkennbaren Fortleben und Fortwirken von $d D$ in der gesamten direkten Überlieferung. Das ungemein heikle Problem der Beeinflussung von $D$ durch einen Lateiner ${ }^{1}$ ist für die Textgeschichte des lateinischen Paulus deshalb nicht von entscheidender Bedeutung, weil die Einheit des lateinischen Textes dadurch nicht nur nicht in Frage gestellt, eher sogar gesichert wird. Denn $d$ kommt als Berater von $D$ nicht in Betracht, allenfalls sein Ahne. v. Sodens Grundsatz aber, jede griechische Sonderlesart von $D$, die sich in $d$ und anderwärts, jedoch nur auf lateinischem Boden findet, als Rückbildung aus dem Lateinischen $z u$ betrachten und deshalb gewissermaßen zu mißachten - da klafft ein tiefer Abgrund zwischen $D d$ und $G g$, wo die Anlehnung an den Lateiner offenkundig ist ${ }^{2}-$ entkleidet in seiner äußersten Konsequenz $D$ seiner Individualität und beraubt den lateinischen Text mit seiner. so ausgeprägten Eigenart seines griechischen Stammvaters. Wie kann es v. Soden verantworten, Lesungen der $D$-Gruppe unter Preisgabe aller anderen Textformen als authentische Überlieferung anzuerkennen, die nach seiner These den Stempel der Willkürlichkeit an sich trüge?

d $g(r)\left(r_{8}\right)$ (gue) vg Ambrst. (m) mit DG gegen die sonstige Gr(iechische Über-

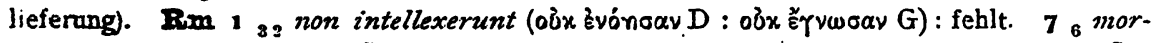

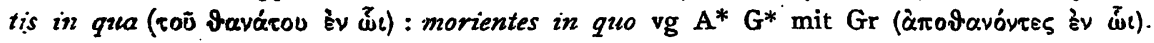

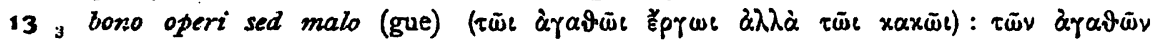

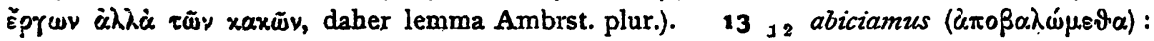

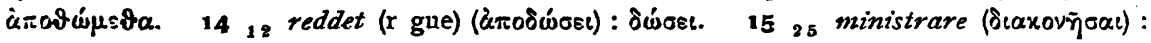
Èsaxovōis (-ñowv). I Cor $16{ }_{12}$ notum uobis facio (notesco $u . \mathrm{g}$ : significo $u$. Ambrst.)

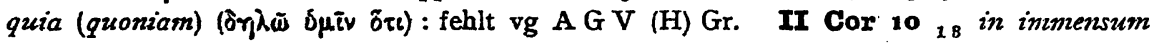

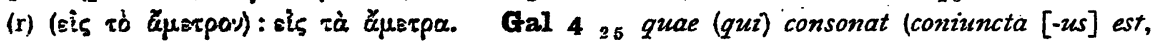

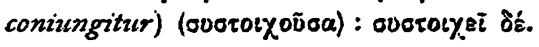

An dieser grundsätzlichen Konstellation wird nichts geändert, wenn offensichtlich dieser oder jener Zeuge sich nachträglich der

1 Siehe $\nabla$. Soden I $1946 \mathrm{f}$.

2 Die Vergewaltigung des griechischen Textes durch den Lateiner in $G$ ist so offenkundig, daB, wo immer $G$ als einziger Grieche mit dem $L t$. übereinstimmt, die lateinische Lesart als aus dem Griechischen nicht bezeugt zu gelten hat. 
griechischen Majorität, wohl auch unter unbewußter Rücklassung einer Spur seiner Vergangenheit, angeschlossen hat:

Im 8 10 si autem Christus.. in uobis fehlte urspr, in d D : fehlt in $\mathrm{g}$ G Ambrst.

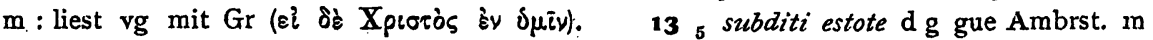

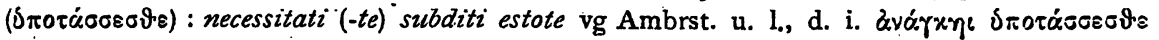

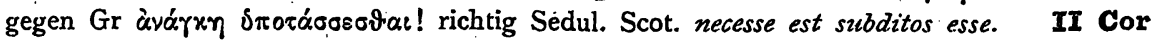

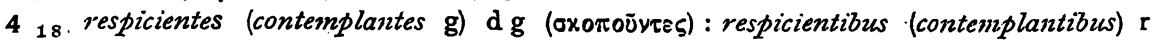

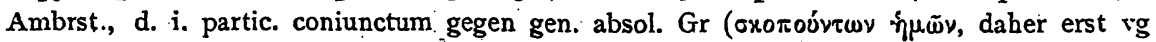
conteimplantibus nobis). 10 ; seruzm (seruus) dg Ambrst. mit D G : fehlt Ambrst. ed. Rom. vg mit Gr. : r verstümmelt. $12{ }_{15}$ diligor $(\alpha \gamma \alpha \pi \omega \tilde{\mu} \mu \mathrm{l})$ : licet . . . diligar vg mit

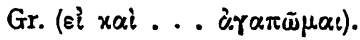

Entbehrt $d$ der Stütze vọn $D$ und aller übrigen Griechen, sofern diese nicht, wie etwa $G$, unmittelbar einem Lateiner angeglichen wurden, so ist der Anschluß aller lateinischen Zeugen an ihn, den Lateiner, um so beweiskräftiger: hier wird mehrfache selbständige Benutzung desselben griechischen Originals zur Unmöglichkeit. Diese völlige Isolierung des $d$-Textes in seinen Sonderlesarten von allen Griechen hätte als Norm zu gelten selbst für die auch aus $D$ allein zu belegenden Sonderlesarten der Lateiner, wenn Soden mit seiner oben erwähnten, von $\mathrm{ihm}$ in ihrer ganzen Tragweite nicht erfaßten These recht hätte.

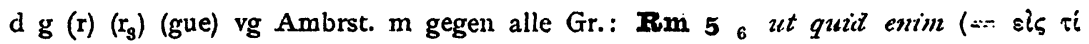

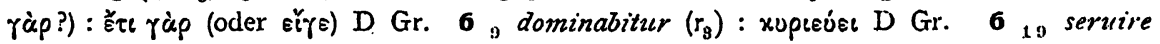

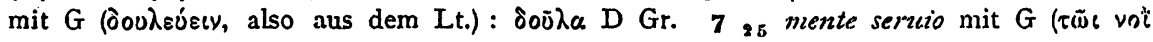

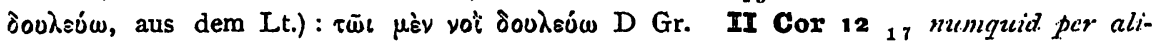

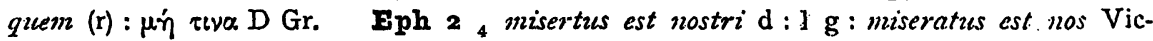
torin. : qua misertus est nostri Ambrst.) (dgl. in I Cor 13,4): qua misertus est nobis

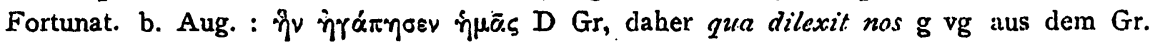

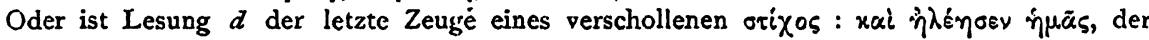
durch homoeotel. ausgefallen?

Diese Sonderbestrebungen des $d$-Typus konnten nicht verborgen bleiben. Es wurden daher Versuche, mehrfach mit ungeeigneten Mitteln, unternommen, der griechischen Überlieferung doch noch zu ihrem Rechte zu verhelfen.

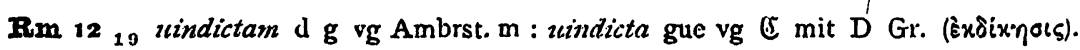

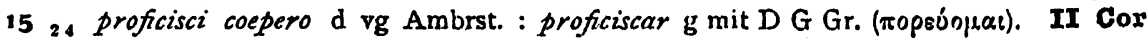
$48 \mathrm{f}$ bieten $\mathrm{d} \times \mathrm{g}$ vg $\mathrm{m}$ gegen $\mathrm{D}$ Gr. grundsätalich (8mal) das Verbum finitum statt des Partizips, das $\mathrm{g}$ als Variante liest, der Amlurst. $7 \mathrm{mal}$ aus dem Gr. einsetzt, Imal (perimus) übersieht, womit er sich verrät. 8 odeinde $\mathrm{d}$ vg Ambrst. : deinde et $\mathrm{g}:$ at $\mathrm{r}$ mit $\mathrm{D}$ Gr (*al). 8 , insuper $\mathrm{d} g$ vg (die meisten) Ambrst. : fehlt $\mathrm{r}$ vg (einige) init D Gr. 13 ॥

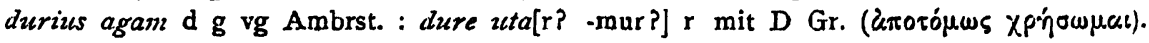

Eingriffe in die lateinische Überlieferung waren gerechtfertigt, wenn es galt, offenkundige Irrtümer des Archetypus zu beseitigen. 
Das konnte mit und ohne griechische Hilfe geschehen, richtig und unrichtig, geschickt und plump. Mißglückte Versuche sind besonders lehrreich, weil sie uns vollwertige Beweise für die unbedingte Abhängigkeit aller vom $d$-Typus liefern. So kehren handgreifliche Fehler in $d$ in allen Texten wieder, teils in Reinkultur, teils verschleiert hinter scheinbar korrekten Lesungen, die meist ohne Befragung einer griechischen Vorlage untergeschoben wurden.

Nicht verbesserte Fehler: Rm $5{ }_{12}$ in quo omnes peccauerunt (statt 'eo quod'

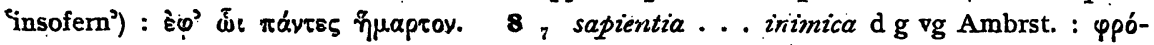

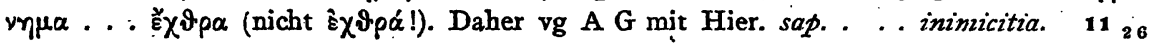

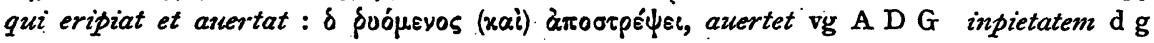
vg Sא Ambrst. : $\alpha \sigma \varepsilon \beta \varepsilon i a$, , danach vg impietates. II Cor 1 , exhortamur (passivisch) :

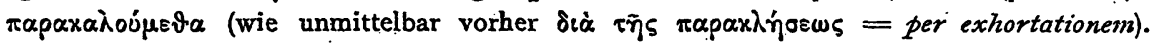

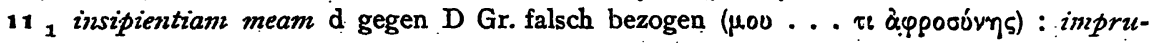

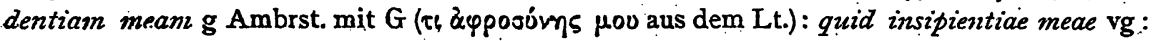

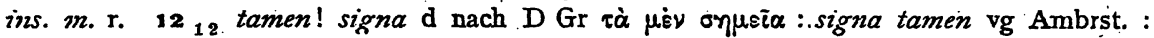

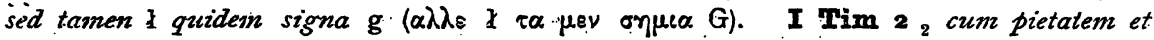

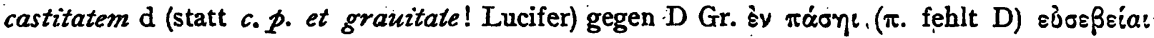

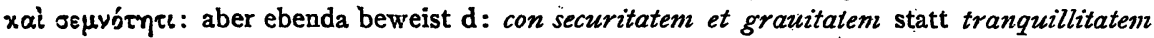

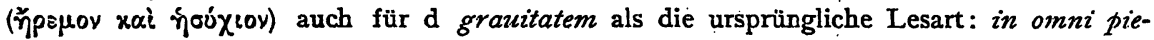
tate $m$ et caritate $m \mathrm{r}$ (acc. aus d) : i. o. pietate et castitate $\mathrm{g}$ 'vg Ambrst. (aus d!).

Nur vereinzelt oder in letzter Instanz verbesserte Fehler: $\mathbf{R m} 2{ }_{15}$ cogitationum accusantium . . . defendentium (genet. absolutus!) d vg: $\mathrm{g}$ u. l. : cogitationibus accusantibus... defendentibus vg D SE Ambrst. 1518 factis d vg Ambrst. : opere g m mit Gr." (êprwt). I Cor $6_{13}$ escae ... . hanc (d. i. uentrem!) . . haec (d. i.

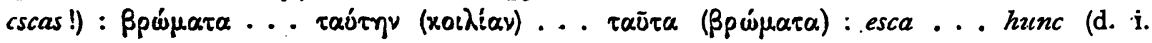
uentrcm) ... haec vg : esca ... hunc... has vg S(C Ambrst. II Cor $10_{13}$ mensus

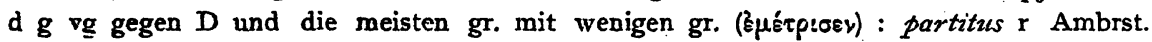

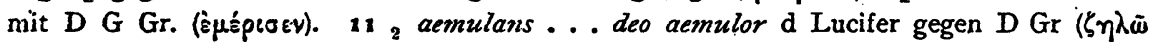

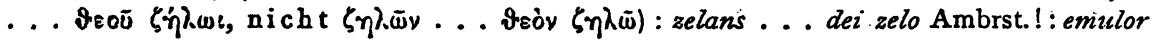
$\$$ zelo ... dei zelo $\mathrm{g}$ : aemulor ... dei aemulatione (aem. dei $\mathrm{r}$ ) $\mathrm{r} \mathrm{vg}$.

Mißglückte Korrekturen: $\mathbf{R m} 4$, tantım manet. $d$ verderbt aus tantum an et

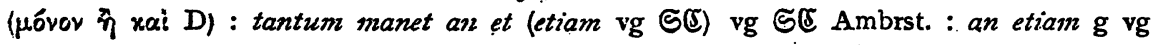

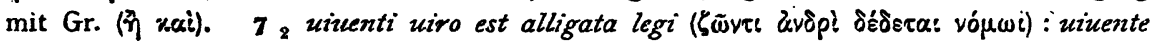
uiro alligata est legi $\mathrm{g}$ vg Ambrst.: man erwartet uiuenti... lege.

I Cor $6_{20}$ ò $\dot{\eta}$ übersetzt nicht d: $\operatorname{man}$ erwartet ergo : et portate $\mathrm{g}$ (gegen G) vg Ambrst. (d. i.

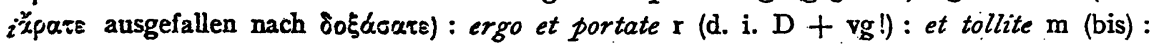

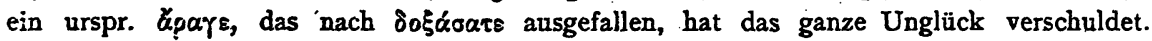

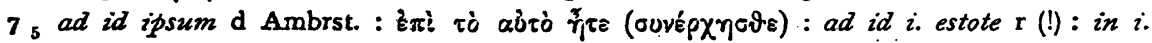
. reuertimini 1 itis $\mathrm{g}(!)$ : reu. in $i$. $i$. vg. $\quad 15_{27} \delta \bar{\eta} \lambda \circ \mathrm{v}$ ö $\tau$ übersetzt nicht $\mathrm{d}$ : sine dubio (nach qui $\mathrm{g}$ gegen $\mathrm{G}$ ) $\mathrm{g} \mathrm{vg}$ : nimirum Ambrst. : manifestum quia $\mathrm{r}$ mit $\mathrm{Gr}$.

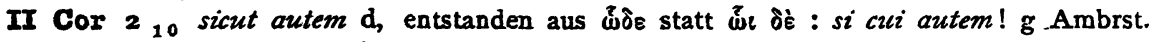
$\mathrm{m}$ : cui autem $\mathrm{rg}$ mit Gr. (⿳亠े冖⿱ ingenium bonum (!) vg: bonum animum Ambrst. : bonum $\mathrm{g}$ : carissimum $\mathrm{r}$ Aug. ${ }_{11}$

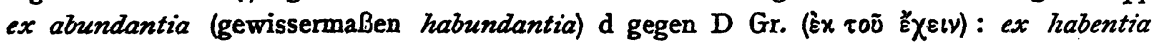
$\mathrm{g}$ ! : ex eo quod habetis vg Ambrst.: $1 \mathrm{~g}:$ ex eo quod quisque habet $\mathrm{r} .1_{12} \cdot{ }_{18}$ conparantes nobis nosmetipsos. non $\mathrm{d}$ mit $\mathrm{D}$ (curxpivovtes éaviois źavtoúc. oठ̉x) : conp. nos-. 
met ipsos nobis. non $\mathrm{g}$ Ambrst. : conp. nosm. ipsos nobis. nos autem non $\mathrm{vg}:$ conp. [sem]etipso[s] sibimetipsis non intelle[gun]t. nos uero non $\mathrm{r}$ Aug. mit Gr. (oüxp. żavtoùs źaucoiss

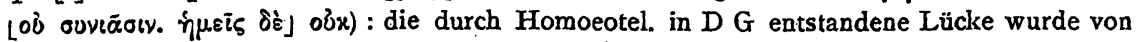
vg teilweise ergänzt, von $x$ aus eignem in Anlehnung an den gr. Text: aber das nicht geänderte in inmenszım erweist $d$ als Urform für das Vorbergehende und Nachfolgende. Denn

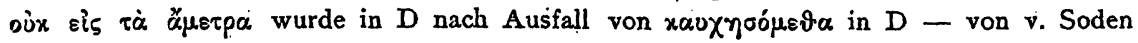

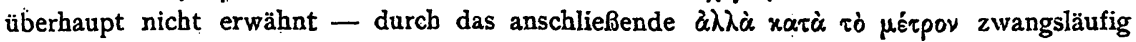

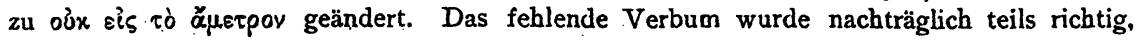

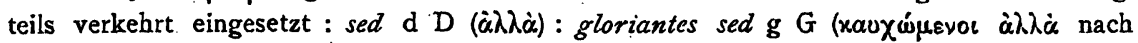

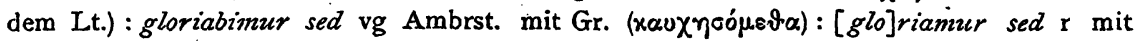

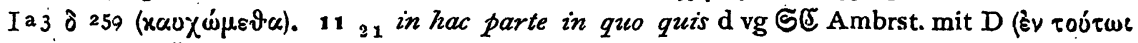

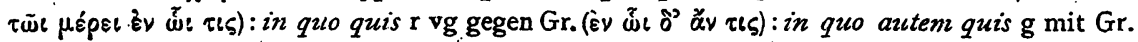
Febr $10_{29}$ deteriora depraecauitur uindictae d gegen D Gr. (Xeipovos à $\xi_{t \omega \vartheta}$

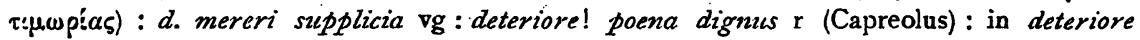

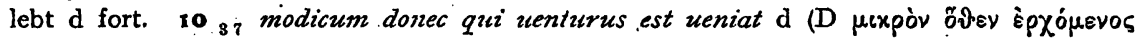
$\eta \xi \xi s)$ : modice donec ueniat $q$. u. e. $\mathrm{r}$ : modicum quantulum (oder ä.) q. u. e. veniet $\mathrm{vg}$ :

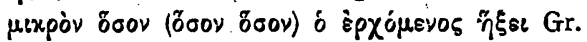

Manchmal wurden vermeintliche Fehler oder Ungenauigkeiten ohne Not beseitigt

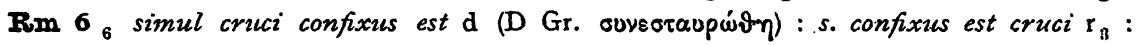
con $1_{\text {asimul }}$ crucifixus est $\mathrm{g}:$ s. crucifixius est vg Ambrst. $\mathrm{m}$ : simul verrät d (vgl. Gal $2{ }_{19}$ ). and confixus ist mit fixus synonym. $15{ }_{28}$ multis iam praecedentibus annis d (àmi

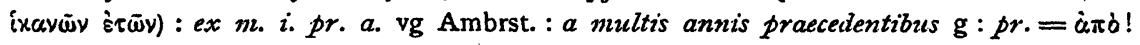

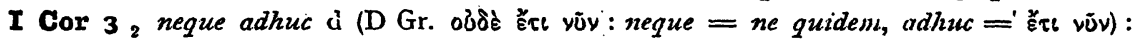
neque adhuc munc $\mathrm{g}$ : neque nunc Ambrst.: nec adhuc quidem $\mathrm{r}:$ nec nunc quidem $\mathrm{vg}$ : neque unverkennbare Reminiszenz an d. vgl, auch II Cor $10_{4} \mathrm{Gal}_{425}$.

Das Bedingtsein aller Texte durch den d-Typus erfährt eine weitere Bestätigung und gewisse Steigerung dadurch, daß abgesehen von gruppenweisen Anschlüsssen an $d$ jeder einzelne Zeuge Sonderlesarten mit $d$ gemeinsam hat, die nur aus direkter Deszendenz zu erklären sind:

1) dg: $\mathrm{r}\left(\mathrm{r}_{\mathrm{B}}\right)$ (gue) vg Vict. Ambrst. $\mathrm{m}$ : Eine Liste von Sonderlesarten $d g$ erübrigt sich: Die Berechtigung der Gruppe Iax 1026 ff (Soden) als einer geschlossenen Einheit bedarf keiner Úberprüfung: Alle anderen Verbindungen aber seien durch einige schlagende Beispiele belegt:

2) $\mathrm{d} r\left(r_{2}\right): g$ vg Ambrst. I Cor 1 , in nulla gratia (Gr.): in ulla gratia $g$ vg: in nullo... aliqua gratia Ambrst. $\mathbf{6}_{2}$ et in uobis (falsch) : et si in uobis. $\mathbf{7}_{2}$ formicationes (Gr.) : fornicutionem (G, aus dem Lt.). 15 s 0 quid et (Gr.) : ut quid et. 15 a2 bestiis : ad bestias. 16 ig Aquila multum (D) : m. A. gr vg : Alquilu Ambrst. II Co1* $1_{16}$ proficisci : transire u. ä. $4_{1 *}$ respicientes (-tibus) : contemplantes (-tibus). ${ }_{4}$ mortale : mortale hoc $\mathrm{g}$ Ambrst. $\mathrm{m}$ : quod m. est $\mathrm{vg}$. $5_{10}$ secundum quod (ea quap): prout. 5 10 amodo : ex hoc: 1 a nunc g. 8 , semper: fehlt, mit Gr. $8_{12}$ promptus

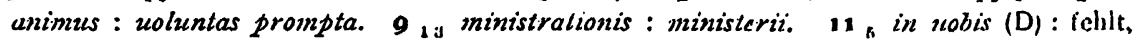
mit Gr. Eph 1 ostenderet : notum faceret (Vict.). $1{ }_{10}$ in dispositione (-em) : I" dispensationem (-ne) (Vict.). Phil 4,7 uerbo uestro (sermone $u_{0}$ ) : rationem (-ne) uestram $(-a) \mathrm{g}$ vg Ambrst. $\mathrm{m}$ : oratione uestra Vict.

Die beiden Klassen $d r_{3}$ und $d$ gue stützen sich auf wenig umfangreiche Teile von $\mathrm{Rm}$ und Gal. $r_{8}$ ist in seiner zweiten Hälfte 
vor allem ein ausgeprägter 'Augustintext', $d$ und $r$ unbedingt ergeben; auch gue-Lesarten, die von der vg abweichen, sind meist ( $2 \mathrm{Imal}$ ) in $d$. begründet. Es liegt in der Natur der Sache, daß Belege für Sonderbeziehungen von $r_{3}$ und gice $z \mathfrak{u} d$ nur Zufallsrecht beanspruchen können. Trotzdem fehlen sie nicht:

3) $d r_{3}: g$ vg Ambrst. (m): $\mathbf{R m} 6_{6}$ cruci confixus est (conf. e. cr.) : crucifixzs est. Gal 4, rursus : denuo (iterum). vgl. 48 .

4) d gue : g vg Ambrst.: $\operatorname{Rm} 14$ g uizoricm et mortucorum (gegen D) : mortuorum et uiuorum vgl. $\mathrm{II}_{36} \mathrm{C}^{12} 2_{2} \mathrm{2}^{\circ}$.

5) $\mathrm{d} \nabla \mathrm{g}: \mathrm{g}(\mathrm{r})\left(\mathrm{r}_{\mathrm{g}}\right)$ (gue) Ambrst.: $\operatorname{Rm} 4_{17}$ deum (Fehler) : eim. $6_{16}$ obauditis (Gr.) : oboedistis. $8_{23}$ gemimus : ingemiscimus (esc-). " I Cor $2_{16}$ Christi gegen D mit den meisten Gr. : domini (r) mit D G. II Cor 4 ss $_{3}$ sicut : secundrim quod (r). $4_{16}$ (is) qui foris est.... (is) qui intus est : exterior... interior (r). $5_{8}$ bonam zoluntatem habentes (habemus $\mathrm{vg}$ ) : consentimus $\mathrm{g}$ Ambrst. : bene arbitranuer $\mathrm{r} . \mathrm{5}_{1}$ g posuit: ponenss

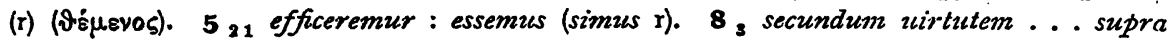
uirtutem : pro uiribus . . . supra uires (u. ä.) ( $\mathrm{r})$. $9_{13}$ in óbaudientiam $(-a)$ : in subiectione (obsequio $\mathrm{r}$ ). $\quad 13_{4}$ infirmi sumus : infirmamur (r). Gal $2_{13}$ duceretur $a b$

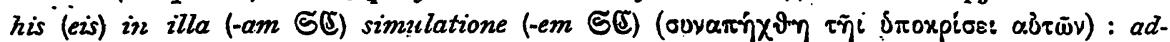
duceretur illorum simulationem $\mathrm{r}$ : simul ductus sit $\mathrm{g}$ (nach $\mathrm{gr}$.) bzw. consentiret simulationi eorum (g) Vict. Ambrst. $4_{20}$ modo : nunc (Vict.). $\mathbf{E p h}_{1}$; sacramentum (I $\mathrm{g})$ : mysterium (r Vict.). Phil 1 18 per occasionem ... per ueritatem : occasione ... ueritate $\mathrm{g} \times$ Ambrst. : sub obtentu ... ueritate Vict. $4_{16}$ in uszum mihi : usibus mcis $\mathrm{r}_{2}$ Ambrst.: $1 \mathrm{~g}$ : in necessitatem incam $\mathrm{g}$.

Die $d v g$ ausschließlich eignenden Lesarten sind ohne Zweifel die bedeutsamsten: hat die Gleichung in der Abstammung der vg, in einer Angleichung und Rückschraubung der $v g$ an den Urtyp oder gar in einer Infizierung des $d$-Textes mit vg-Lesarten ihren Grund? Für die letzte äuberst gewagte Lösung, der Soden das Wort redet, finde ich keine sichere Unterlage. Man könnte allenfalls II Cor $4_{16} .5_{8}$ Phil $I_{18}$ diese Erklärung in Erwägung ziehen: in den beiden ersten Fällen wird die Autorität von $d$ durch Lucifer, seinen Kronzeugen, gestützt, Phil $\mathrm{I}_{18}$ durch Cyprian und Tyconius. $\mathrm{Daß}$ wir auf die Vermittlerrolle des Hieronymus verzichten können, wird unten gezeigt. Die Deszendenz der $v g$ von $d$ ist nicht $z \mathrm{u}$ bestreiten.

6) d Vict. : g vg Ambrst.: Gal $22_{2}$ inter gentes : in gentibus. $2_{14}$ ingrediuntur: ambulant (-arent, incederent). 4 g ut cognouistis : cum cognoueritis $\mathrm{vg} \mathrm{m}$ : cognoscentes

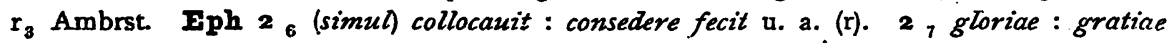
(Gr.). vgl. Gal. $3_{21} \cdot 6_{16}$ Eph $I_{19^{\circ}}$

7) d Ambrst.: $g(r)\left(r_{8}\right)$ (gue) vg (Vict.) (m): Rm. $8_{28}$ procedunt : cooperantutr. $8{ }_{36}$ morte adficimur : mortificamur $(\mathrm{m})$. $13_{4}$ male (2mal) : malum (2mal) (gue $\mathrm{m}$ ) in eum : ei gue $\mathrm{vg} \mathrm{m} .16_{2}$ in quibuscumque desiderauerit uestri gegen $\mathrm{D}$ Gr. : in quocumque negotio uestri indiguerit $\mathrm{\nabla g}$ mit Gr.: in quocumque uestri desiderauerit $\mathrm{g}$. 16 g meum gegen D Gr. : nostrum $\mathrm{g}$ vg (Gr.). I Cor 15 14 uacua : inanis (r). 1525 omnia enim suliecit sul pedilus eius vor ${ }_{28}$ : dass. nach ${ }_{26} \mathrm{~g}$ r vg. $15_{31}$ domino (D): 
Christo lesu domino nostro (r). 15 s $_{9}$ hominis ... pecoris (m S) (D) : hominum ... pecorum (r m rec. Flor.) (Gr.). II Cor $2_{3}$ a quibus : ex his a quibus (de quibus) (r). $7{ }_{12}$ ut manifestetur (-aretur) sollicitudo nostra : propter (ad) manifestandam sollicitudinem (industriain) nostram (zestrain) ( $\mathrm{r}) \cdot{ }_{18} \mathrm{10}_{18}$ (et) probatus est : ille probatus est $\mathrm{r}$ .vg : 'ille est probatus g. $12{ }_{15}$ et superinpendam (D) : fehlt $\mathrm{r} g$ vg (Gr.). Gal 310 quotquot : quicumque (quaec) (r Vict.). Phil $1_{4}$ orationcm : deprecationem (r). I Tim $2{ }_{3}$ salútari : saluatore $(\mathrm{r})$. ${ }_{2}{ }_{7}$ magister $(\mathrm{l} \mathrm{g})$ : doctor $(\mathrm{r})$.

8) $\mathrm{d} \mathrm{m}: \mathrm{g}$ ( $\mathrm{r}$ ) $\mathrm{gg}$ Ambrst. (Vict.): $\boldsymbol{R m} 15_{13}$ pace : pace in credendo (r). I Cor $2{ }_{10}$ scrutat . . . alta $($ alta $\mathrm{l} \mathrm{g})$ : scrutatur . . . altitudines (-em) $\mathrm{g} \mathrm{r}$ : scrutatur . . profunda vg Ambrst. $6_{16}{ }_{17}$ se iungit (iiingit se) 2 mal ( $(\mathrm{g})$ : adhaeret (r). $7_{31}$ habitus: figura. $16_{18}$ confortamini agite uiriliter (ziril. ag.) : uiriliter agite (et) confortamini $(\mathrm{r})$. Gal $3_{\text {в }}$ perficiamini (-emini) : consummamini (-m-) (r Vict.). $3_{19}$ factorum gratia gegen

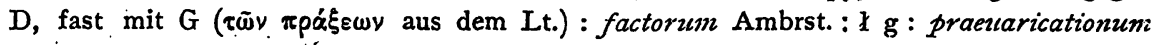
$\mathrm{g}$ : transgressionis $\mathrm{r}:$ propter transgressiones (-em) $\mathrm{g}$ Ambrst. ed. Rom. I Tim $6_{1}$ habeant: arbitrentur (existiment) ( $\mathrm{r}$ ). $\mathbf{6}_{8}$ uestitum : tegumentum (-a) (quibus tegamur) (r).

In diesen Stadien der Überlieferung hat ein der Textgestalt von $d D$ wesensfremder Grieche die Entwicklung des Lateiners nicht beeinflußt.

Die offensichtliche, überragende Autorität des $d$-Textes wird dadurch nicht. erschüttert, daß sich die Vertreter der direkten Überlieferung vielfach gegen $d$ zusammenschließen. Meist handelt es sich um eine einheitliche Gegnerschaft, deren einigendes Band nicht in eigentlich 'neuen' Lesungen besteht, sondern in synonymen Wörtern und Konstruktionen: Substantiven, Adjektiven, Pronomina, Präpositionen, Konjunktionen, Partikeln, Adverbien, Tempora, Modi und Wortstellungen. Die indirekte Überlieferung steht bald $\mathrm{zu} d$, nicht minder häufig aber auch - und zwar seit Iren. Tert. Cypr. auf der Gegenseite.

Der Prioritätsstreit ' $d$ oder die anderen' läßt sich nicht nach einer allgemeinen Formel oder einem apodiktischen Werturteil zu Gunsten von $d$ oder der indirekten Zeugen entscheiden. Und doch löst sich das Problem durch die Tatsache, daß $d$ durch seine primitiven Wörter, Wortbildungen und Wortgefüge vielfach überrascht: diese Erkenntnis hat rückwirkende Kraft: auch bei scheinbar gleichwertigen Synonymen - das Vorlegen langer Listen im einzelnen erübrigt sich hier - gebührt $d$ die Vorhand:

I) Substantiva und Adjektiva: II Cor $5_{10}$ saeculum : mundum. $8_{\text {s }}$

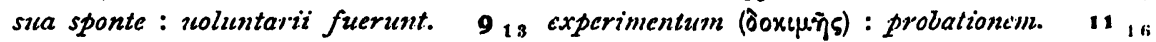

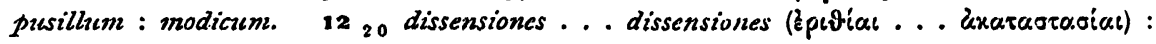
dissensiones ... seditiones. Gal $2{ }_{2}$ gentilibus : gentibus. $\mathbf{E p h}_{1}$ " in nationem filiorum (els vioveoiav) : in adoptionem $f_{0} 1_{21}$ intium (apy $\eta$ !) (Iren.) : principatum.

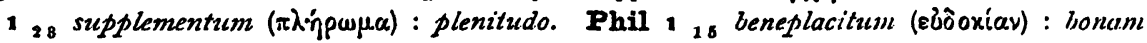

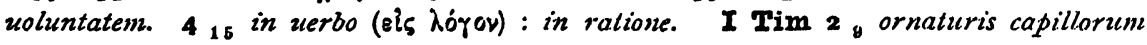

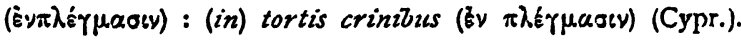


2) Konjunktionen, Partikeln, Adverbien: Gal 3 19 quoadusque : ḋonec (m). Eph $2_{19}$ iuxta (Iren.) : prope (Vict.).

3) Verben: II Cor 5 10 ferat (xopiontal) (Lucifer) : referat (recipiat, reportet).

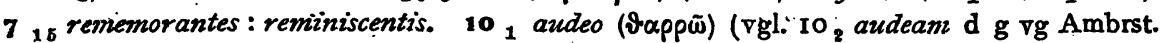

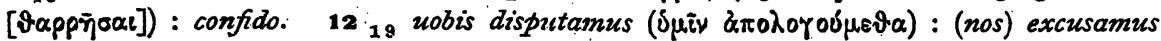
(excusemus) nos apud uos (uobis). Iph $2_{\text {g }}$ coexcitauit. (ovv'ýreıpev) (Hil.) : simul excitauit $\underline{r}_{-}$: con- (simul) suscitautit (res-). collocauit (vgl. Vict.) (Hil.) : (simul) consedere (sedere) fecit. I Thess I s declaratus ( $\xi \xi \eta \dot{\eta} \chi \eta \tau \alpha c)$ : diffamatzs (innotuit g). I Tim 1 so disciplinam accipiant : discant (erudiantur g) (Hil.). $5_{22}$ serıa (ińpec) (Lucifer) : custodi. $6_{7}$ efferre ( $\varepsilon_{\xi \varepsilon v e \gamma x \in i \nu)}$ : auferre (Cypr.). Hobr $6_{6}$ recrucientes

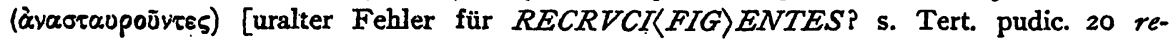
figentes crici] : rursum crucifigentes $\mathrm{vg}$ : denuo configentes . . . cruci $\mathrm{r}$.

4) Verbalformen: I Cor $16_{22}$ siat : sit. II Cor $10_{17}$ gloriatur $^{2}$ d. i.

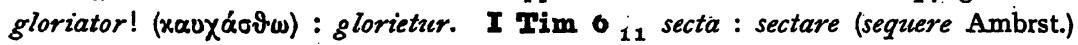

Diese immerhin rein äußerlichen Unterschiede treten noch stärker in die Erscheinung, wenn sich die Zeugen in der Abkehr von $d$ zwar einig sind, untereinander aber wiederum in Gruppen sich zusammenfinden, oder dieser und jener als Einschichtiger ein Sonderleben führt:

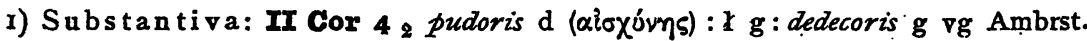
$\mathrm{m}$ (Tert.) : confusionis $\mathrm{r}$ (Aug.). 7. i $1_{1}$ in negotio d (mit den meisten Gr.) : negotio g vg

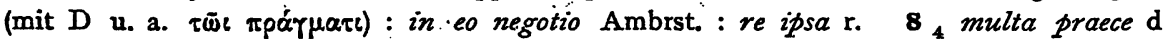

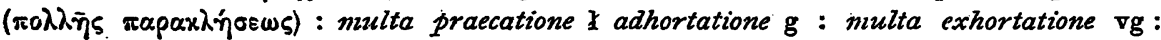

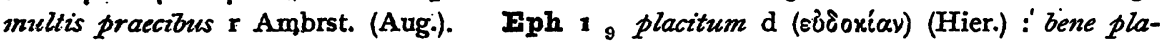
citum g vg SE Ambrst. : bonum placitum $\mathrm{vg}$ : bonam uoluntatem $\mathrm{r}$ : uoluntatem Vict.

2) Adjektiva: I Cor 6 g inpudici d : fornicarii vg Ambrst. m (Iren. Cypr.) :

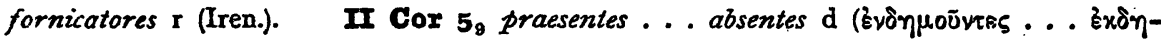

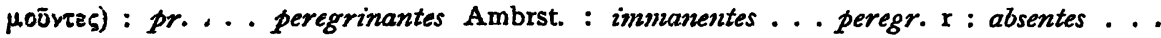
praesentes $\mathrm{g}$ $\mathrm{g}$ (Tert. Lucifer).

3) Konjunktionen, Adverbien: I Cor $6{ }_{8}$ neque d ( $\mu \dot{\eta}_{\imath \iota} \gamma \varepsilon$ ) : nedum $\mathrm{r}$ : quanto magis $\mathrm{g}$ vg. II Cor $4_{16}$ et si d ( $\varepsilon i$ rai) (Tert. Ambrst.) : licet $\mathrm{vg}$ : et licet si

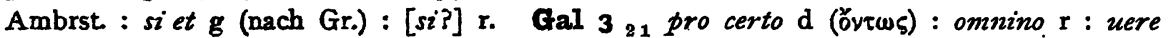

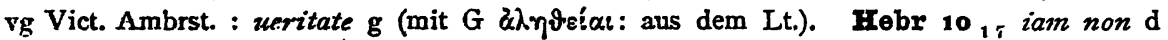

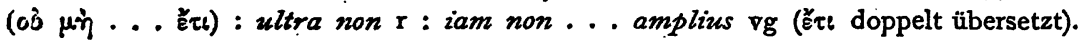

4) Verben und Partizipien: I Cor $7_{8}$ manserint $d$ ( $\mu$ sívwoเv) : maneant $\mathbf{v g}$ :

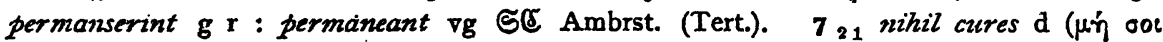
$\mu \equiv \lambda \hat{\varepsilon} \tau \omega)$ : non tzbi sit (s.t. Ambrst.) curae $\mathrm{g}$ vg Ambrst. : non ad te pertinet $\mathrm{r} .1528$

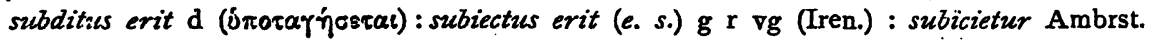

I g. II Cor $4_{\mathrm{g}}$ coopertum d : opertum $\mathrm{g}$ vg : obuelatum $\mathrm{r}$ : om Ambrst. m $\mathrm{S}$. $91_{0}$ subministrauit (d. i. -bit) d (Aug.) : ministrabit $g$ Ambrst. : $\alpha$ tribuet $g$ : praestabit vg (Cypr.) 10 , obsecro d : rogo g vg Ambrst. : praecor $r .11$, portaret is d: sustineretis $\mathrm{r}$ vg Ambrst. (Lucifer) : pateremini g. Gal $2_{19}$ cruci confixus sum $\mathrm{d}$ : confixus sum cruci $\mathrm{r}$ vg Ambrst. : crucifixus sum $\mathrm{g}$ Vict. (vgl. $\mathrm{Rm} 6_{6}$ ). I Thess $1_{8}$ sit

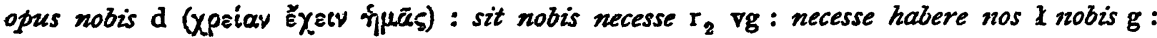

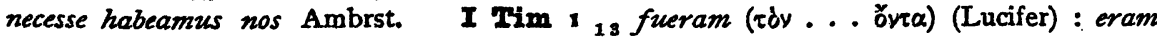

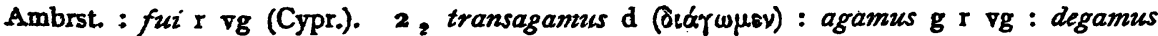

Zeitscbr. f. d. neatest. Wiss. 20. Band xg21. 
Ambrst. $2{ }_{15}$ salua autem fiet $\mathrm{d}$ (Hier.) : 1 img $\mathrm{g}$ (fiat) : salua autem erit $\mathrm{r}$ Ambrst. $\mathrm{m}$ : saluabitur autem $\mathrm{g}$. $\mathrm{g}$. perseuerauerint $\mathrm{d}: \mathrm{l} \mathrm{g}:$ permanseri( $n) t \mathrm{r} \mathrm{g}$ $\mathrm{g}:$ manserit Ambrst. : maneat $\mathrm{m}$.

5) Stilistisches und Syntaktisches: Rm $6_{14}$ in uos ... dominabitur

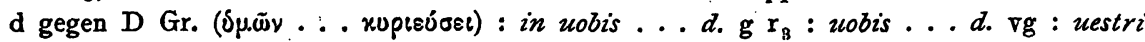
...d. Ambrst. II Cor $7_{14}$ quae ad Titüin fuit d ( $\mathrm{vg}$ : apud T. Ambrst. $8_{5}$ semet ipsi d : semet ipsos $\mathrm{g}$ vg Ambrst. : se ipsos $\mathrm{r}_{1} \cdot 1_{2}$ fiduciam $\mathrm{d}:$ per $f . \mathrm{g}:$ p. eam. f. $\mathrm{r}$ Ambrst. : p. e. confidentiam $\mathrm{vg} .10_{12}$ intra nos $\mathrm{d}$ :

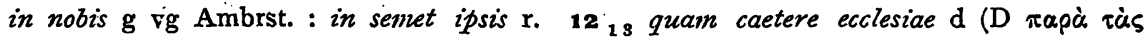

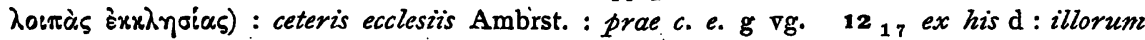

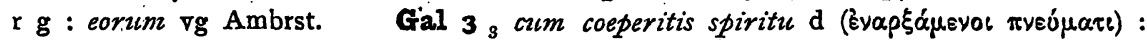
cum sp. c. $\mathrm{m} \mathrm{S}:$ : ut cum sp. c. $\mathrm{r}$. $\mathrm{g}$ Vict. : incipientes sp. g Ambrst. $3_{2}$ q quae postea retegebatur $\mathrm{d}: 1 \mathrm{~g}$ ( $q$. p. renelaretur $\mathbf{g}$ : quae futura erat ut reuelaretur Vict. : quae incipiebat reuelari Ambrst. : quae reucelanda erat $\mathrm{vg} . \quad \mathbf{E p h} 1_{15}$ audiens uestra fide . . et caritatem d

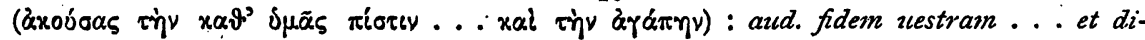
lectionem $\nabla \mathrm{g}$ : audita et fide uestra ... et caritatem $\mathrm{g}:$ aud. $f . u . \ldots$ et dilectione Vict.

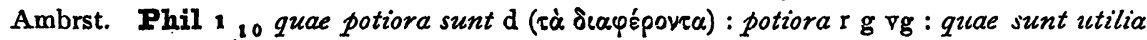
Ambrst.

$\mathrm{Daß} d$ und nur $d$ die ursprüngliche Lesart bietet, lehren auch hier prächtige Beispiele:

I) Substantiva: II Cor $4_{17}$ secundum excellentiam in sublimitate d (xav' $\delta \pi \varepsilon \rho \beta 0 \lambda \eta \eta \eta v ~ \varepsilon i s ~ \delta \pi \varepsilon \rho \beta 0 \lambda \eta \dot{\nu})$ : supra modum in sublimitate vg Ambrst. : s. m. in sublimitatem $\mathrm{g}$ (nach Gr.) : iuxta incredibilem modum et in incredibilem modium $\mathrm{r}$. $9_{10}$ in

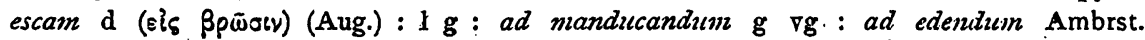

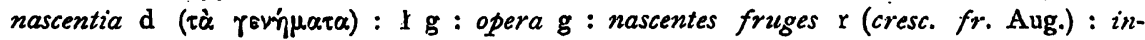
crementa frugum vg (Cypr.): fructum Ambrst.

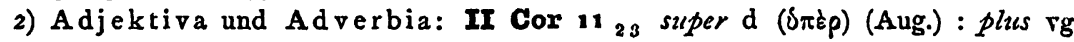

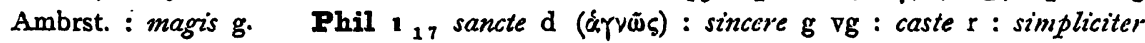
Ambrst. : sancte et caste Vict. (d $+r$ !).

3) Verben und Partizipien: IT Cor 11,3 statui d (íprooáunv) (Lucifer) : aptabi $\mathrm{r}$ (Aug.) : paraui Ambrst. : desponsaui (Hier. Aug.) : 1 spopondi $\mathrm{g}$ : despondi vg

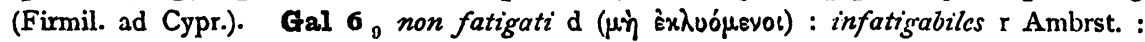

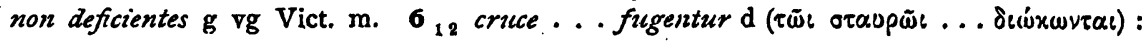
per crucem persecutionem ...patiantur $\mathrm{g}:$ in criccem ...p....p. $\mathrm{r}:$ crucis . . p.

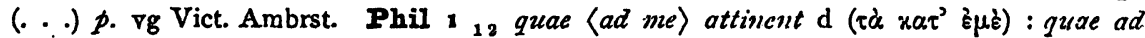
me pertinent Ambrst. : (ea g) quae circa me sunt $\mathrm{g} \mathrm{vg}:$ quantum arl me $\mathrm{r}$ : quae ad me viell. Urform. I Thess $1_{8}$ exiuzit $\mathrm{d}(\bar{\varepsilon} \xi \varepsilon \lambda \hat{\eta} \lambda \cup \vartheta \vartheta v): 1 \mathrm{~g}:$ profecta est $\mathrm{r}_{2} \mathrm{vg}:$ processit Ambrst.

Auch diese Entwicklungsphase des Paulinischen Textes setzt Befragung eines abseitsstehenden griechischen Zeugen keineswegs voraus. Im Gegenteil. Manche Sonderlesung wäre nicht entstanden, wenn ihr Urheber griechische Originale eingesehen hätte. Dies freie und willkürliche Schalten mit dem lateinischen Wortlaut hat schließlich dazu geführt, daß alle Lateiner nicht nur $d D$, sonclern die gesamte griechische Überlieferung preisgaben: 
d D Gr. gegen die übrigen Lt.: Bm 8 .8 wocati sunt (Lucif. Aug.) : is s. sancti: $11{ }_{11}$ offenderunt (richtig) : sic 0 . (desgl. die indirekte Überi.) (falsch I). ${ }_{12}{ }_{1}$; bona : bona non tantum (solum g) coram deo sed etiam (gue $m$ ) (desgl. die gesamte indirekte

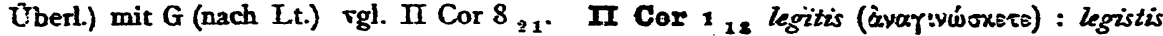
11 in omni (Ev aavi) : in onznzbus (desgl. indirekte Ūberl.). Gal 2 is credidimzus

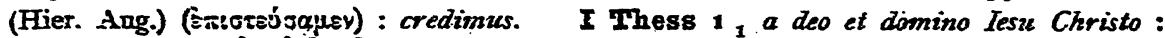
fehlt $\left(r_{y}\right)$-mit $\mathrm{H}$ ò I j 6. $78 \mathrm{G}$ (aus $\mathrm{Lt}$.).

Der bisherige Verlauf der Untersuchung hat drei Tatsachen festgelegt: I) Der $d$-Typus lebt fort in der gesamten direkten Überlieferung. 2) An eben diesen Archetypus fesseln jeden einzelnen Zeugen persönliche Beziehungen. 3) Alle Texte haben gemeinsame von $d$ abweichende Lesungen, die nicht, wie allenfalls ausgemerzte Fehler, alle insgesamt die eine von der anderen unabhängig geworden sein können.

Daraus folgt, daß der $d$-Typus, wie er sich uns darstellt, einer Redaktion oder Revision unterzogen wurde, die in den bisher vorgelegten Fâllen vornehmlich stilistischer Natur war und auf das griechische Original kaum Rücksicht nahm.

Ehe die Frage nach der Existenz des überarbeiteten $d$-Typus und nach seinem wirklichen Namen - ich nenne ihn vorlāufig $d_{1}$ aufgeworfen und beantwortet wierden kann, müssen Wirken und Schaffen des Redaktors, also seine Art, möglichst genau ergründet werden.

Bedingt und gefordert war eine Überprüfung des Archetypus zunächst im Interesse der Beseitigung augenfälliger Versehen. Diese sind meist gleichlautend in allen direkten Quellen beseitigt worden, vielfach allerdings, wie oben gezeigt wurde, nicht ohne daß die Urform Spuren hinterlieb (s. S. 108f.).

Nicht minder wichtig als die Ausrottung offenkundiger Fehler und Irrtümer war die Beseitigung gewisser Graezismen, mit welchen sich ein Kenner des Griechischen und des griechischen Urtextes allenfalls abfinden mochte, nicht aber die lateinisch denkende Masse:

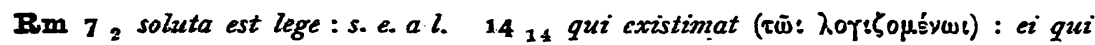

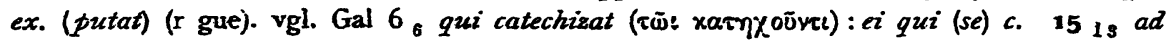

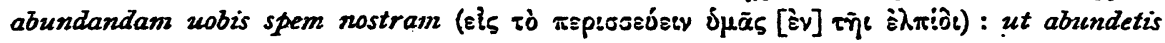

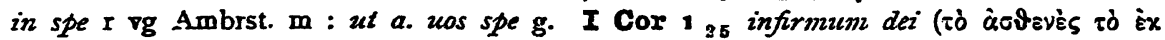

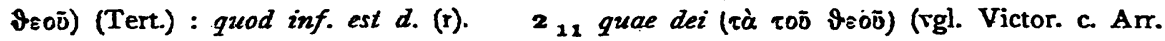
$\mathrm{I}, \mathrm{II}): q$. d. sunt $\mathrm{r} \mathrm{rg}$ Ambrst. : in deo $\mathrm{g}$ (daher $\mathrm{G}$ ) : in deo sunt $\mathrm{m}$. ${ }_{12}$ spiritum

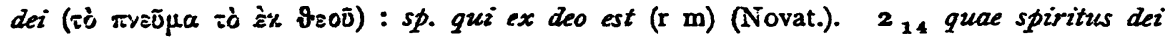

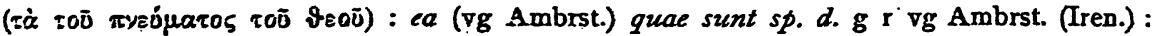

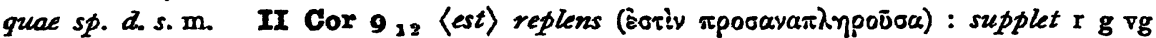
Ambrst. : k est adsupplenda g. 12,0 inueniar uobis : inu. $a$ u. (r). Gal 4 g qui...

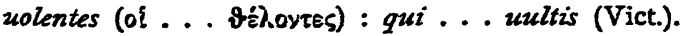


Manches Wort, manche Redewendung und Konstruktion war veraltet, wurde wohl auch als ungenau und unlateinisch empfunden und mußte dem modernen Sprachgebrauch angepaßt werden. Das Fremdartige aber verrät, wo das Ursprüngliche liegt (s. S. 112. I 13 f).

Der Eingriff aber in die Überlieferung wurde wiederum zur Notwendigkeit, wenn es galt, einen lateinischen oder griechischen Ausdruck durch den erst im Laufe der Zeit erstarrten griechischen oder lateinischen Terminus technicus zu ersetzen:

I Cor 1 10 scissurae : scismata g $r$ vg Ambrst. und alle indirekten Zeugen, vgl.

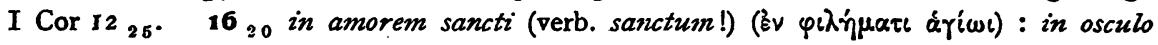
sancto. Gal $4_{24}$ (aliud ex alio〉 significantia ( $2 \lambda \lambda \eta \eta$ үороó $\left.\mu \in v a\right)$ : in allegoria $\mathrm{r}_{3}$ : per allegoriam dicta $\mathrm{g}$ vg Ambrst. vgl. Aug. trin. 15.

Die große Bedeutung primitiver Strukturen und Wörter für die Bewertung von $d$ und des $d$-Typus ist einleuchtend. Das Werden des $d$-Textes liegt jenseits der Zeit, da die Termini allegoria, osculum, schisma, selbst euangelium allgemein wurden, die $d$-Form gehört einer Epoche an, da man noch von amor, scissura, selbst von adnuntatio sprach und sprechen konnte, dà man sich der Abkürzungen $d s$, $d i$ usw. für deus und für dominus bediente. Diese Erkenntnis sichert dem $d$-Text die überaus willkommene, von Rechts wegen zu fordernde zeitliche Überlegenheit. Jeglichen Zweifel aber an der Vormachtstellung von $d$ bannt die Tatsache, daß rudimentäre Lesarten, welche $d$ aus den Zeiten des ersten Versuches einer Verdolmetschung des griechischen Urtextes bewahrt hat, aus der indirekten Überlieferung - abgesehen etwa vom Irenaeus latinus, von Tert., Lucifer, der selbst Fehler aus dem $d$-Typus übernommen hat - zum Teil gar nicht $z \mathrm{u}$ belegen sind, während $d_{1}$ vielfach von. zahlreichen Autoren zitiert wird. $\mathrm{Rm}$ i I 11 hat $d_{1}$ (gegen $d D$ Gr.) durch Einschaltung von sic den Finalsatz (iva) in einen Konsekutivsatz verwandelt. Die Erweiterung $\mathrm{Rm} 12{ }_{17}$ bona + non tantum coram deo sed etiam durch $d_{1}$ aus II Cor $8_{21}$ haben alle indirekten Zeugen ebenso gedankenlos übernommen, wie den Fehler I I $_{11}$.

$\mathrm{DaB}$ es der Urheber von $d_{1}$ nicht bei pflichtmäßigen Änderungen bewenden ließ, sondern überlieferte Wörter und Wendungen ohne Not durch Synonyma ersetzte, lehren zahlreiche Beispiele. All dies geschah noch ohne Rücksichtnahme auf eine $D$ wesensfremde griechische Vorlage. Das Interesse für den Lateiner ließ ihn sogar manchmal wohl unbewußt die Grenzen seiner Befugnisse, welche durch einen griechischen Text schlechthin gezogen waren, überschreiten (S. $114 \mathrm{f}$.).

Der Einbruch keineswegs vereinzelter Lesarten in $d_{1}$, welche 
$D d$ fremd, in anderen griechischen Hss aber heimisch sind, berreist, daß der Schopfer von $d_{1}$ sich auch mit griechischen Vorlagen beschäftigt hat. Aber selbst hierdurch wird der $d$-Typus keineswegs seines Charakters als Archetypus aller Hss entkleidet. In diesem Zeichen stehen $d . D$ vielfach allein gegen die gesamte lateinische und restliche griechische Überlieferung:

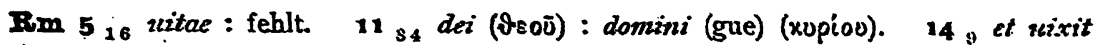

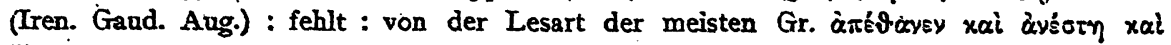

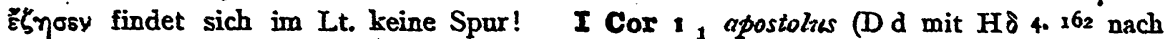

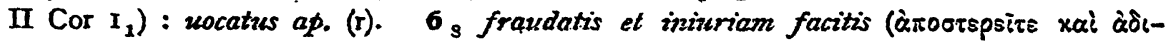

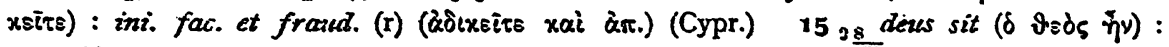

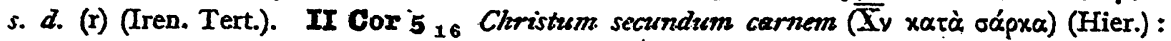

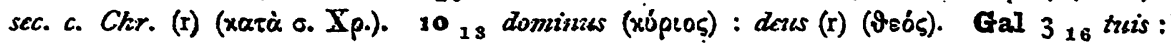

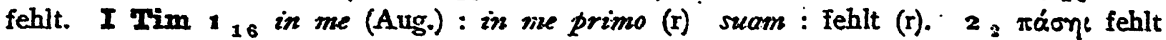
D d .(Lucifer) : omani (r). Hebr $\mathbf{7}$ is constituzit sacerdotes homines (xavioryouv ispsis

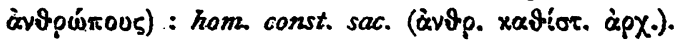

Wie $z u$ erwarten, kennen die indirekten Zeugen, mehrfach in großer Zahl, fast nur die für $d_{1}$ geforderten Lesarten.

Bald erfreuen sich beide der Unterstützung der meisten oder zahlreicher Griechen, während die Gegenpartei entweder geschlossen zusammensteht:

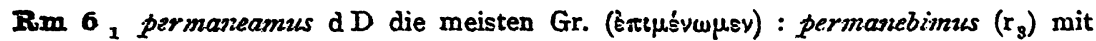
vielen Gr. $9_{32}$ legis d D viele Gr. : fehlt, desgl. in den meisten Gr. II Cor $11_{14}$

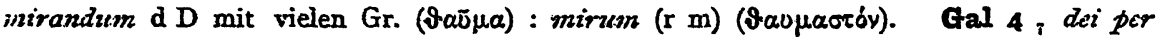
(.hristum d D mit vielen Gr. : per deum ( $\mathrm{r}_{3}$ Vict.).

oder in verschiedene Lager gespalten bald dieser, bald jener griechischen Fassung Gefolgschaft leistet. Bei dieser Konstellation fällt es schwer, zu entscheiden, welche Textform bei einer Drei- oder Vierteilung $d_{1}$ zuzusprechen ist:

I Cor $6{ }_{5}$ sapiens d D mit einigen Gr. (oọós) : quisquam s. $\mathrm{r}_{1} \mathrm{~g}$ Ambrst. mit

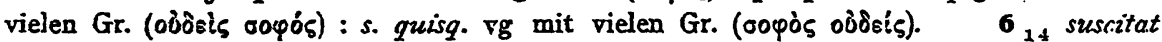

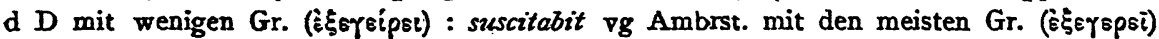

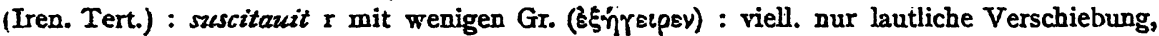
wie $\operatorname{Rm} 6{ }_{15}$ peccamus d D : peccabimus vg Sહ Ambrst. mit vielen Gr. : peccauimus

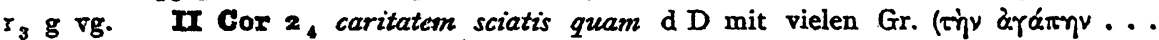

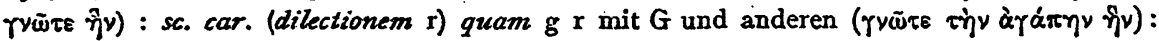
sc. (cognoscatis Ambrst.) quam car. $\mathrm{rg}$ Ambrst. (latinisiert). $10_{10}$ inquid $\mathrm{d} \mathrm{D}$ mit den meisten Gr. : inquiunt $\mathrm{g}$ vg mit $\mathrm{H}^{1}{ }^{1}$ syr.: aiunt $\mathrm{I}$ : fehlt Ambrst. $10_{12}$ nos vor aut d D : nach comparare $\mathrm{g} \times \mathrm{vg}$ mit den meisten Gr. : nos ipsos nach comp. Ambrst. 11 \&

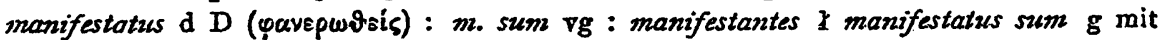
$\mathrm{G}$ und anderen Gr. (

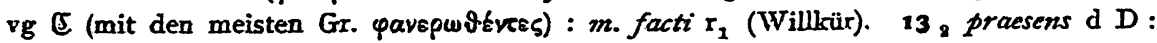
ut pr. vg Ambrst. mit den meisten Gr. : quasi pr. g. $13{ }_{11}$ pacis et caritatis d D mit einigen Gr. : p. et dilectionis $\mathrm{Vg} \subseteq\left(\mathbb{S}\right.$ Ambrst. : pacis $\mathrm{g}$ mit $\mathrm{GF} \mathrm{H}^{48}$ : dilectionis et 


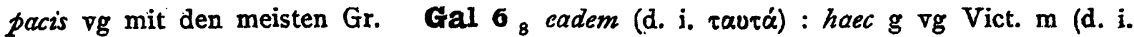

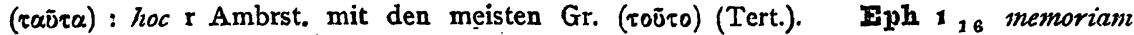
faciens d D mit einigen Gr. : m. f. uestri g G : m. $u . f . \mathrm{r}$ vg Vict. mit den meisten Gr. : commemorationem u. facere Ambrst. . Phil ${ }_{14}$ logui dei d D : dei loqui vg Ambrst. mit einigen Gr. : domini logui $\mathrm{g}$ G : loqui $\times$ mit den meisten Gr. I Thess

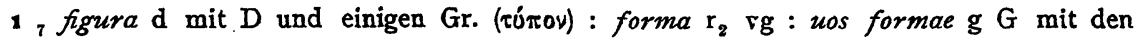

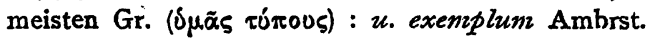

Wo indirekte Zeugen. Auskunft geben, entscheiden sie sich meist für die revidierte Textform. Kenntnis des Urtypus verraten wiederum nur ganz vereinzelt Irenaeus und Tertullian.

Soweit wir Werden und Wesen von $d_{1}$ zu erlauschen vermögen, wurde die Isolierung von $d$ vornehmlich durch Ausmerzung von Fehlern und Graezismen, veralteter bzw. mißverständlicher Wörter und Wendungen, Beseitigung wirklicher und scheinbarer stilistischer Unebenheiten bedingt, die sich zuweilen bis zur Vergewaltigung des gesamten griechischen Urtextes steigerte. Vergleichung und Nutzbarmachung griechischer Texte spielen nach Ausweis der Listen S. II f. eine durchaus untergeordnete Rolle.

Sein oder Nichtsein dieses revidierten $d$-Textes in unserer Zeit hängt von der Beantwortung der Frage $a b$, ob zur Klärung aller nicht rein zufälliger Beziehungen von $g, r\left(r_{2}\right), r_{3}, g u e, v g$, Vict., Ambrst., $m$ untereinander einer der genannten Zeugen berufen ist oder nicht. Daß sie alle denselben Vater oder Großvater und Stammvater hatten, steht fest, und es wäre wohl denkbar, daß ei n em unter ihnen alle verpflichtet waren. Doch nur unter folgenden Bedingungen:

I) Es dürfen diesen Text mit $d$ keine Sonderlesarten verbinden, während die übrigen Zeugen untereinander einig sind.

2) Es dürfen sich die einzelnen Zeugen auch nicht. gruppen- und kreuzweise gegen ihren Führer stellen.

3) Sonderlesarten, insbesondere Fehler und Irrtümer dieses bevorzugten Zeugen müssen, wenn auch in bescheidenem Ausmaß, in den abhängigen Texten nachweisbar sein.

Diesen Forderungen wird kein Hs-Typus, kein Exzerptor, kein Kommentator gerecht. Im Gegenteil. Die S. I Ioff behandelten Gruppierungen $d g, d r, d r_{3}$ usw. sind über jeden Zweifel erhaben; die Vertreter der Gegenpartei bilden grundsätzlich eine geschlossene Einheit. Paarungen und Kreuzungen von $g, r, v g$ usw. untereinander sind an der Tagesordnung, insbesondere von $g, r\left(r_{2}\right), v g$, Ambrst., von $r\left(r_{2}\right)$ und allen anderen, von $r_{3}$, gue, vg, Ambrst., von gue, $v g, m, S$, der vg und aller übrigen, des Ambrst. und Victorin. Auch 
größere Gruppen fehlen nicht mit scharf ausgeprägten Eigenarten. Nirgends, in schroffem Gegensatz zu $d$, wuchert oder lebt ein Makel oder Merkmal eines Zeugen bei allen übrigen fort.

$\mathrm{Da}$ somit kein lebender Zeuge als Vermittler namhaft zu machen ist, war $d_{1}$ ein Codex eignen Rechts, der, wenn auch heute verschollen, die Brücke schlug vom reinen $d$-Typus hinüber zur gesamtēn jüngeren direkten Überlieferung. Nicht nur der direkten. Wenn, wie bereits S. II5. I16. II7 gezeigt wurde, Belege für altertümlichste Lesungen von $d$ in den indirekten Quellen mit seltenen Ausnahmen fehlen, dafür aber die jüngere Lesung $d_{1}$ allenthalben, zum Teil schon bei Cyprian, begegnet, so kann es nicht mehr zweifelhaft sein, daß schon die ältesten christlichen Autoren $d_{1}$ einsehen konnten und sogar grundsätzlich benutzt haben. Galt es, den Archetypus zu vernehmen, bot sich einem Hier., Aug. usw. ebenso wohl Gelegenheit wie uns. Daß der Urtypus nicht ausstarb, beweisen der erst im VI Jh. geschriebene zweisprachige Claromontanus und dessen um Jahrhunderte jüngere Tochterhss. sowie $G F$ aus dem IX Jh. Und wer immer überhaupt nur den Archetypus gelten lassen wollte, wie Lucifer u. a., dem war auch dies unbenommen.

Die Datierung von $d_{1}$ ist zunächst an das Werden des $d$-Typus gebunden, der seinerseits an $D$ gekettet ist, wie $D$ an das gleichnamige Evangeliar. Dieses aber hüteten nicht ohne Grund die Mönche vom hl. Irenaeus zu Lyon als wertvollsten Schatz, und man wird $D$ nicht leichten Herzens vom Namen des großen Apologeten trennen. Darüber hinaus können wir feststellen, daß $d_{1}$ entstanden sein muß: 1) vor dem frühesten Auftreten der $g$ - und $r$-Formen, 2) vor $m$, 3) vor der $v g$ des Hier. (um 385), 4) vor der Zeit, da der sog. Ambrst. und Vict. schrieben, d. i. vor 380 bzw. 350. Durch diese Erkenntnis wird der $d$-Typus selbst spätestens in den Anfang des IV Jh. gestellt.

Die späteren Schicksale von $d_{1}$ sind von der Textgeschichte der Paulinischen Briefe nach der indirekten wie direkten Über. lieferung unzertrennlich. Diese gilt es vorerst $\mathrm{zu}$ verfolgen.

Der Urheber von $d_{1}$, welcher neben stilistischen auch textkritische Änderungen, wenn auch in beschränktem Maße, vornahm, löste dadurch de facto den Lateiner von seiner griechischen Vorlage. $\mathrm{Ob}$ der Lateiner erst durch das Eingreifen des Redaktors sui iuris wurde, oder ob die Befreiung schon früher erfolgt war, ist mit Sicherheit nicht $z u$ entscheiden. Die grobe Wahrscheinlichkeit aber spricht für einen ursächlichen Zusammenhang beider Vorgänge: Wer den Lateiner selbständig machte, revidierte und redigierte ihn 
auch, und er hat sichtlich die Übersetzung a uch mit dem Original noch einmal konfrontiert. Dadurch wird das Entwicklungsproblem des lateinischen Textes wesentlich vereinfacht. $D$ as $Z$ wischenund Bindeglied $d_{1}$ wäre nichts anderes, als der erste lateinische Paulus, welcher der griechischen Stütze entbehrte: die selbstverständliche und notwendige Stufe im Leben und Werden jedes Teiles der lateinischen Bibel; die Stufe, für welche die S. I 8 formulierten Bedingungen durch. $d$ ipso facto erfüllt sind. Die Trennung vom Griechen, welche einen Abschied auf Nimmerwiedersehen, eine Entfremdung auf immerdar bedeutete, schuf freie Bahn für ungehemmte Entwicklung, für ein Eigenleben des lateinischen Textes.

Von $d_{1}$ lösten sich die ältesten Vertreter der $g-, r_{-}\left(r_{2}-\right), r_{3}-$, gue-, vg-Formen, die letzten Vorlagen des Victorin, Ambrst. und von $m$. Jeder einzelne sagte sich ohne Wissen des anderen von $d_{1}$ los, und ein jeder ging eigne Wege. Hieraus erklären sich die relativ großen Verschiedenheiten der einzelnen Texte in ihrem Verhalten zur gemeinsamen Vorlage: weitgehende Emanzipation bedingt ebensowenig spätere Datierung, wie aus engerer Anlehnung höheres Alter der betreffenden Quelle gefolgert werden dürfte: sie alle waren einander koordiniert, nicht nach- oder untergeordnet. Es blieb jedem einzelnen unbenommen, unter Befragung und Ausnutzung griechischer Texte und Entlehnungen bei lateinischen Stammesgenossen sich sein ihm passend erscheinendes Gewand anzulegen, wodurch er seine Abstammung bald mehr, bald weniger verschleierte.

Es ist daher schief und ein aussichtsloses Unterfangen, nach der griechischen Vorlage von $r_{,} r_{3}, g u e, v g, m$, des Victorinus und Ambrst. Ausschau zu halten, als ob es neben den $D E G F W$-Codd. je eine $R$ GVE VG $M$-Gruppe usw. gegeben hätte. Nicht minder verfehlt war es, sich zu wundern oder zu freuen, weil die Freisinger Hs 'zum ersten Male eine zusammenhängende, zweifellos vorhieronymianische Übersetzung brachte, welche nicht zur Erläuterung eines anderen Textes, sondern lediglich um ihrer selbst willen geschrieben wurde' (Ziegler S. 30). Es gab für alle nur eine, und zwar die griechische Vorlage, dieselbe wie für $d, g$, w, den Archetypus des griechischen Claromontanus. Hierfür liefern die Interlinearübersetzung $g$ und der 'vulgarisierte' $f$ den besten Beweis, und würde uns die bereits i. J. 1483 verschollene 
griech.-lat. Hs. der Paulusbriefe, die im XII Jh. in Bamberg lag (s. Bresslau Neues Archiv 2 I, I896, I45 [nr. 48] I74ff) wiedergeschenkt, sie könnte diese Erkenntnis nur bestätigen. Wer nicht dem $d$ Typus Gefolgschaft leistete, hatte das Anrecht auf eine griechische Vorlage verwirkt.

Dafür haben die Deszendenten von $d_{1}$ von dem Vorrecht freier Entfaltung ausgiebigen Gebrauch gemacht, und bei den bereits erwähnten Paarungen und Kreuzungen (s. S. I18) vermögen wir vielfach weder aus inneren, noch aus zeitlichen Gründen zu entscheiden, wer der Gebende, wer der Nehmende ist. Unmittelbare Abhängigkeit eines Zeugen vom anderen ist überhaupt ausgeschlossen. Sonderlesungen jedes einzelnen im Gegensatz zu $d$ und allen übrigen Quellen erschweren obendrein jede Untersuchung. Im Einzelfall mag sogar zweifelhaft sein, ob die Gleichung $g=r, g=v g, g=$ Ambrst. usw. auf früher oder später Übertragung von hüben und drüben oder umgekehrt beruht. Denn es besteht die große Masse der Sonderlesungen jener Gruppierungen zweiten Grades in synonymen Wendungen u. dgl.; deren doppeltes Auftreten nicht in ursächlichem Zusammenhang stehen $\mathrm{muB}$. Auch da, wo die Urheber der $r-\left(r_{2}\right), r_{3}-$, gue-, vg-, Vict.-, Ambrst.-, m-Typen griechische oder lateinische Texte konsultiert haben, wie wir es noch heute in $g$ sehen, mußten Streichungen, auch wenn sie ein Zeuge unabhängig von einem anderen vornahm, durchwegs $z u$ demselben Resultat führen. Und endlich konnten in Einzelheiten, im Gegensatz zu der allenthalben vorherrschenden $d$-Fassung; auch zwei und mehr Redaktoren jeder für sich auf denselben Ausdruck, dieselbe Konstruktion verfallen sein. Aber trotz alledem bleibt ein so bedeutender Bestand an kongruenten Lesungen übrig, daß Beziehungen und Annäherungen zwischen den einzelnen Abkömmlungen von $d_{1}$ nicht $\mathrm{zu}$ leugnen sind. Unter Verzicht auf Vorlegung des umfangreichen und durchaus einwandfreien Materials sei in Kürze über die Beziehungen der einzelnen direkten Zeugen untereinander, bei $r$ auch zur indirekten Überlieferung, berichtet.

Marius Victorinus stützt sich in seinen Erläuterungen $z u$ Gal und Eph grundsätzlich auf $d(g)$ und $v g$, die Lemmata im PhilKommentar, der jünger ist als der $z u \mathrm{Eph}^{1}$, übertrug er meist selbst aus dem Griechischen (s. o. S. 104). Er kennt den $r$-Typus ${ }^{2}$ und

1 S. zu Phil 2 6ff p. 1207 b.

2 Vict. $=r:$ Eph $I_{0}$ mysterium (Ambrst.). I 11 sortem consecuti sumus. Phil $i_{1}$; contumaciasn. ebda non sancte neque caste Vict. : non sancte $\mathrm{d}:$ non caste $\mathrm{r}$. 
die' diesem verwandten oder befreundeten $r_{3}$ und gue-Formen ${ }^{1}$. Zahlreiche Sonderlesarten, mögen sie auf eigner Übersetzung ${ }^{2}$, auf Entlehnung ${ }^{3}$ oder Willkür ${ }^{4}$ beruhen, oder wie immer entstanden sein - es fehlt auch nicht an Entgleisungen ${ }^{5}$ - sind, abgesehen von Nachwirkungen bei dem sog. Ambrosiaster (s. u.), für die Geschichte des Paulustextes wirkungslos verklungen.

Der Ambrosiaster war Eklektiker und hat mit allen Textformen unverkennbare Berührungspunkte. Griechische Codd. hat er eingesehen und sich nutzbar gemacht. Belastet aber wird der Text durch Vorliebe bzw. Abneigung des Verf. für bzw. gegen gewisse Worte und Wendungen (s. o.), wodurch das Bild der von ihm benutzten Hss (bzw. Hs) noch weiter verzerrt wird. Endlich verrät er in den Lemmata aus Gal und Eph Beziehungen zu Vict., ohne daß man ihn als Abschreiber entlarven könnte ${ }^{6}$. Daß der Ambrst. nahm und nicht gab, beweist die Tatsache, daß seine persönlichen Sympathien und Antipathien in der früheren und späteren Überlieferung abgesehen von mittelbaren oder unmittelbaren späten Entlehnungen in $g$, (f) und von seiten des Sedulius Scotus spurlos untergegangen sind.

Das ps.-Augustinische Speculum (m) lebt in zwei Rezensionen fort, der rec. Sessoriana (S) und Floriacensis (FMVLC), von denen letztere, außer an $d$ und $v g$, sich enger an $r$ anlehnt: vielfach erweckt der Text den Eindruck willkürlicher Gestaltung.

$g$, in seinem Grundstock längst erkannt als ein mehrfach reinerer $d$-Typus, als selbst $d$ es ist, ist in seinen primären und sekundären Lesungen obendrein vom $r$-Typus, von der vg, dem Ambrst. unzertrennlich. In $g$ stößt man auch auf Lesungen, welche dẹn Werken

1 Vict. $=\mathrm{r}_{\mathrm{n}}$ : Gal $4 \mathrm{x} 6 \mathrm{ff}$ praedicans (g). ebda bonum est aemulari (cí. d). Vict. = gue: Vict. adu. Arr. 1, $37: \mathrm{Rm}$ II ${ }_{35}$ reddetur (m).

2 Vgl. S. 105 und Anm. 4 dieser Seite.

${ }^{3}$ Phil I 8 certamen et agonein Vict. : certanen d vg : agonem Tert. (Vict. = $\mathrm{d} \mathrm{rg}+$ Tert. wie oben (Anm. $2=\mathrm{d}+\mathrm{r}$ ), wie Phil 2 , it haesitationibus uel detractationibus $=\nabla g+d)$. Gal $6{ }_{11}$ ecce mit Hil.

4 Adu. Arr. $\mathrm{x}, 24$ : Col $\mathrm{I}_{15}$ ante omnem creaturam (statt omnis creaturae). ${ }^{16}$ in terris (in terra), constituta (creata). I 1 ante ommia (ante omnes), consistunt (con. stant). I \& quae est principium (qui est pr.), fierct (sit), primarius (primogenitusi. I 20 per ipsum reconciliare et reconuertere (per eum reconciliare).

- Phil $2{ }_{10}$ ornnes genua fectant, caelestium, terrestrium et infernorum!

${ }^{6}$ Ambrst. = Vict.: Gal $2{ }_{21}$ non sum ingratus gratiae dei. Eph $2{ }_{1}$ ut exhiberst et reconciliaret. $2_{19}$ incolae. $4_{81}$ auferatur. $5_{11}$ obizergate. verdüchtig ist Gal I 23 solummodo Ambrst. : tantummodo Victorin., seiner Eigenart entsprecbend (s. S. 105) statt tantum. 
des Cyprian, Tertullian u. a. entnommen wurden. Sedulius Scotus wird von Traube (s. o.) als Schreiber des Cod. genannt, kein Wunder, wenn der Text der Paulinischen Briefe beider nahe, wenn auch im einzelnen nicht geklärte Beziehungen aufweist. Dies gilt auch von Gilda. Werden und Entwicklung von $g \mathrm{zu}$ ergründen wäre eine dankbare, aussichtsvolle Arbeit. Das erstaunlich tiefe geistige Nivéeau dessen, der $g$ das individuelle Gepräge verlieh, verrät sich in unmöglichen Wortbildungen, wie usitantibus = roîs oṽov (II Cor I $_{1}$ Phil I ${ }_{1}$ u. ö.), commei $=$ of oìv żoí (Phil $4{ }_{21}$ u. sonst), $e x$

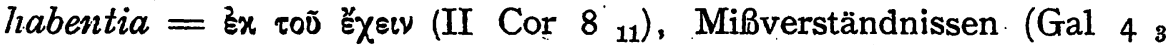

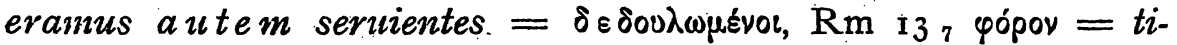
morem), Konstruktionen, Wortstellungen (autem, enim am Satzanfang) sonderbarster Art.

Der $r$-Typus; dargestellt durch $r, r_{2}$, gründet sich auf $d$. und $v g$ und ist, soweit dies möglich, aus einem Gußß. Ohne $d$ und $v g$ sînd die mehr relativ als absolut schwerwiegenden und folgenschweren Sonderlesarten von $r$, das Produkt eignen Lebens und Erlebens, nicht $z u$ verstehen. Nur zu geringerem Teil sind sie entstanden auf Grund vorheriger Befragung eines griechischen Codex, dem sich der Schöpfer - von $r$ manchmal auf seltsame Art anpaßt:

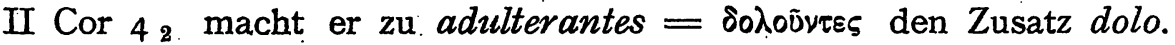
Würde uns ein Zufall den Codex in die Hand spielen, den der Urheber des $r$-Typus eingesehen hat und den gewiß keine hochwertigen neuen Lesarten zierten - einen Cod. $R$ hat es natürlich nie gegeben (s. S. 120) - seines Nimbus wäre $r$ mit einem Schlage entkleidet: und das hat er ob seiner Willkür wohl verdient.

Nichtsdestoweniger war dieser Paulustext, in $g$ und $m, r_{3}$ und gue, bei Marius Victorinus und dem Ambrst. bereits nachweisbar, das Handexemplar zahlreicher Kirchenväter und Kirchenschriftsteller. Der vornehmste, aber keinesfalls der erste unter ihnen war Augustinus, der den $r$-Text in allen seinen Schriften mit einziger Ausnahme des Speculum bald mehr, bald weniger ausschließlich zitiert.

Sichere Spuren des $r$-Textes finden sich bei Hilarius von Poitiers ( $\dagger$ 366), dem Donatisten Tyconius (IV Jh.), bei dem. Spanier Priscillian, der als erster Ketzer 385 in Trier hingerichtet wurde, in den Schriften des Ambrosius, der Bischöfe Optatus von Mileve $\left(\dagger^{-} 385\right)$, Chromatius von Aquileia ( $\dagger_{407}$ ), Paulinus von Nola ( $\dagger_{43}$ ), Nicetas von Remisiana in Dazien ( $\dagger$.um 450), des Presbyter Sedulius (V Jh.), in den Briefen des Papstes Gelasius (492-496), in den Werken des Fulgentius, Bischofs von Ruspe ( $\dagger$ 533) und des kar- 
thagischen Diakons Fulgentius Ferrandus ( $\dagger$ um 550) und endlich bei Gregor von Tours $(\dagger .594)$ und dem Lyoneser Diakon Florus (IX Jh.).

Ausschließlich oder doch grundsätzlich zitieren den $r$-Typus der Laie Heraclianus in seiner Disputation mit dem Bischof Germinius von Sirmium im Jahre 366, im Anfang des V Jh. die Bischöfe Possidius von Calama in der Vita Augustini, Petilianus von Cirta auf dem Konzil von Karthago vom.Jahre 4II, Aurelius und Capreolus von Karthago, Euodius von Uzel in Numidien, Cerealis von CastelloRipensis in Mauretania Caesariensis, die afrikanischen Bischöfe im Geleitbrief zum Libellus emendationis des gallischen Mönches Leporius, sodann der jüngere Arnobius, Ps. Sulpicius Seuerus, endlich der Historiker der Vandalenverfolgung, der Bischof Victor Vitensis, und Papst Agapitus I (535/36) in seinen Briefen. Die Listen beweisen, wie dringend wir einer kritischen Sonderausgabe der Bibeltexte der bedeutendsten christlichen Autoren benötigen.

$r_{3}$, gleichfalls aus $d$ und $v g$ bestehend, ist seiner Eigenart nach auch in seinem ersten Teil ( $R m)$ durchaus ein Augustintext, wenn auch nicht so reinen Gepräges wie im Galaterbrief, wo der $r$-Typus unverkennbar ist. Sonderlesarten hatten keine Bedeutung bei der Nachwelt.

Dasselbe gilt von den wenigen Eigenarten von gue, der ebenfalls $d(g)$ und der $v g$ seine Gestalt verdankt, daneben auch dem $r$ Typus nicht fern stand, ohne ihm blind ergeben gewesen zu sein. Die Verbindungen mit Beda waren wohl indirekter Natur, sie erklären sich aus der vg und Augustinus.

Endlich die Vulgata. Wäre dieser Typus erst von Hieronymus um das Jahr 385 (Hier. epist. 27) geprägt worden, dann konnte die $v g$ die älteren Texte des Victorinus und Ambrst. ebensowenig beeinflussen, wie den gleichfalls früheren $\boldsymbol{r}$-Typus. Man müßte vielmehr die $v g$ als das Becken ansehen, in das Rinnsale aller Überlieferungsquellen zusammenströmten. Für die Erklärung der Gleichung $d=v g$ wäre dann sogar die künstliche Rückbildung einer entarteten Textform zum $d$-Typus durch Hieronymus zu erwägen, auch wenn er es nicht ausdrücklich bezeugt hat. Und der gelehrte Kirchenvater hätte fürwahr mit richtigem Gefühl bei der Bearbeitung der Paulinischen Briefe aus dem Urquell geschopft.

Nun trifft aber wider Erwarten gerade das Gegenteil zu. Ein $v g$-Typus war, ehe alle anderen wurden, außer $d$ und $d_{1}$. Von ihm und durch ihn wurden nicht nur früher, sondern auch mächtiger 
als von jedem anderen Quell der direkten Überlieferung die übrigen Texte durchsetzt und befruchtet ${ }^{1}$, nachdem er sich selbst vom $d$-Typus bzw. von $d_{1}$ losgesagt hatte. Denn die vg-Form ist und bleibt für das Verständnis aller Textformen unerläßlich. Daß etwa allen Zeugen erst nachträglich, aber unmittelbar nach ihrer Geburt, strich- oder tropfenweise die vg eingeimpft worden wäre, kann ernsthaft nicht in Rechnung gesetzt werden.

Es gebührt also einem Text vom Schlage der vg nächst dem verschollenen $d_{1}$ das Hauptverdienst oder die Hauptschuld an der Entwicklung und Verwicklung der verschiedenen Überlieferungszweige. Ihr erstes Auftreten bei Victorinus fällt unbedingt in die Mitte des IV Jh. Wer "weiter vordringen will, muß die indirekten Textzeugen zur Aussage aufrufen.

Auch ohne weitausĥlende Erörterung der indirekten Überlieferung der Paulinischen Briefe, ohne eingehende Deutung aller wertvollen und wertlosen Varianten in schier ungemessener Zahl, deren Ursprung und Fortleben, können die Tatsachen erkannt und gewürdigt werden, daß Lucifer von Cagliari (vor 358), der $d$ an wichtiger Stelle (s. S. jog o. zu I Tim $2_{2}$ ) in reinerer Gestalt las, von Sabatier mit Fug und Recht zur Rekonstruktion von $d$ Hebr 413 herangezogen wurde, daß auch Faustinus von Rom (um 370), Tyconius, der Donatist $u$. a. m. einen weniger fehlerhaften $d$-Text kannten und benutzten; dab Commodians ${ }^{2}$ Anspielungen auf Paulus, so dürftig sie auch sein mögen, sich mit $d$ tatsächlich decken, daß dem Römerzitat im Barnabasbrief ${ }^{3} d$ durchaus entspricht.

Die zahlreichen Exzerpte und Belege eines Cyprian und Tertullian aus Paulus lassen den heutigen Text ahnen, der für Tertullian noch oder gar wieder in weiterer Ferne liegt als für Cyprian. Die Übereinstimmung beider mit noch heute $z u$ uns redenden Zeugen fällt, abgesehen von wenigen Ausnahmen, gewiß nicht zufälliger-

1 Alle Erörterungen über 'Hieronymus und die $v g$ ' sind müßig und fruchtlos, so lange nicht endlich einmal festgestellt wird, welche Sonderlesungen der $v g$ nur bei Hier. und seit Hier. nachweisbar sind.

${ }^{2}$ Commod. instr. 2, 9. I9 I Cor 13 eramen ut sonans : eramentum sonans $d$ : aes sonans vg. apol. 1046 I Thess $4_{17}$ obuiam Christo mit d D : obuiam domino vg mit den übrigen Gr. Diese Zitate Commodians stammen nicht aus Cyprian, der diese Verse in seinen Testimonia überhaupt nicht ausgehoben hat (gegen B. Dombart Commodianus und Cyprians Testimonia Z. f. w. Th. 22 [1879] 374 ff.).

${ }^{3}$ Barnab. epist. interpret. ed. Heer 6,2 p. 41 $\mathrm{Rm} 9_{3 \mathrm{~s}}$ et qui crediderit in illum : et qui crediderit in eo d : et omnis qui credidit in eum $\mathrm{vg}$. 
weise zusammen mit der Kongruenz $d$ vg. Viele Beispiele sind den entsprechenden Bänden des Corp. Scr. Eccl. Lat. (3. 20. 47) leicht zu entnehmen. Die Paulusexemplare des Tertullian und Cyprian hatten ebensowenig untereinander engere, allen anderen Texten fernliegende Berührungspunkte - s. S. I04 Anm. - wie etwa mit $d$ im Gegensatz zur vg oder umgekehrt. An diesem Befund ändern auch gelegentliche Annäherungen an $d^{1}$ oder an die $v g^{2}$ nichts. $d v g$ erscheinen noch als eine Einheit, mag sie im $d$-Typus oder in der Zwischenstufe $d_{1}$ begründet sein. Für Tertullian und Cyprian war der Schnitt zwischen $d$ und $v g$ noch nicht vollzogen. Freilich kennt Tert. adu. Marcion. und sonst bereits eine berühmte Variante einiger $v g$-Hss. zu I Thess $4{ }_{17}^{3}$ : aber eben diese Variante stützt sich auf die gesamte griechische Überlieferung außer $D G$.

Im Gegensatz zu den beiden Afrikanern zitieren Hilarius von Poitiers ${ }^{4}$ und Ambrosius von Mailand ${ }^{5}$ sowohl den $d$-Text als auch die vg: die Gewissenhaftigkeit des Ambrosius unterscheidet sich vorteilhaft von der lässigern Art des Hilarius. Beide bekennen sich naturgemäß grundsätzlich zum $d$-Typus. Hierdurch aber wird die Tatsache nicht berührt, da $\beta$ auch nach Aussage der indirekten Zeugen um die Mitte des IV Jh. beide Textformen eignen Rechtes nebeneinander standen.

Diese nunmehr doppelt gesicherte Erkenntnis erhält eine überraschende Erweiterung dadurch, daß der lateinische Irenaeus nicht minder als Novatianus, der sich rühmte, ein assertor euaingelii

1 Einer der krassesten Fälle für Bevorzugung von d von seiten des Tert. wäre adu. Marc. 5, 3 Gal $2{ }_{3}$ Graecus coactus est mit d (und zwar von rechtsivegen, weil coacturs der Urübersetzung eigen war): gentilis compulszes cst vg; für $\mathrm{C} y \mathrm{pr}$. testim. 3,78 Tit. 3 то quoniam peruersus est hutiusmodi et peccat et est (cum sit d) a semetipso damnatus mit d : quia subuersus est qui eiusmodi est et delinquit cum sit propric izudicio condemnatzes vg.

2 Bevorzugung der vg bei Tert. adu. Marc. 5, 7 p. 595 I Cor $8_{6}$ nobis tamen mit vg:

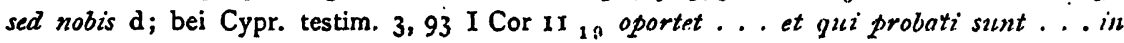
uobis mit vg : op. enim...et probati ... inter uos d.

8 Tert. adu. Marc. 3, 24 p. 42 I. 5, 15 p. 628 obuiam donino mit vg, de carn. res. 24 p. 59. 41 p. 86 obuiam Christo mit d D.

${ }^{4}$ Hil. $=\mathrm{d}$ : in ps. 124, 4 Eph $2{ }_{8}$ coexcitauit et conlocautit (colligauit falsch d) : conresuscitauit et consedere fecit vg. Hil. $=\mathrm{vg}$ : in ps. $142,12 \mathrm{Rm} 8{ }_{28}$ pro notis postulat (post. pro nobis vg) gemitibus inenarrabilibus : postulat gemitibus qui eloqui non possunt $\mathrm{d}$.

5 Ambr. $=$ d: de Jacob 1, 15 p. I4 Rm $7{ }_{13}$ mors est . . pareat . . milit operatum est mortem : factum est mors . . apparcat . . operatum est miki mortem vg. Ambr. $=\mathrm{vg}:$ de Jacob $\mathrm{I}, 19$ p. 9. $19 \mathrm{Rm} 6_{17}$ in eam formam doctrinae in quam (qua u. 1.) traditi estis : in quam traditi estis formam doctrinae d. 
et Christi. zu sein, die Paulinischen Briefe, und zwar mehrfach ein und denselben Vers, bald nach dem $d$-Typus, bald nach der $v g$ zitieren: bei Irenaeus ${ }^{1}$ herrscht $d$ vor, bei Novatian ${ }^{2}$ halten typische $v g$-Lesarten den $d$-Fassungen beinahe die Wage. Würden nicht Zitate aus dem AT und den Evangelien auch für Novatian die it als Grundtext fordern, es. wäre in Anbetracht der zahlreichen Belege für $d=v g$ schwer zu entscheiden, ob Novatians Handexemplar ein $d$ - oder ein $v g$-Typus war, ob die $d$-Fassungen als Rudimente des Urtypus oder die vg-Lesarten als Modernismen zu bewerten sind. Das aber lehrt Novatian mit Bestimmtheit, daß der vg-Typus, d. i. eine im Sinne der $v g$ durchgeführte Recensio des $d$-Textes um die Mitte des III Jh. sich nicht nur vorbereitete, sondern bereits abgeschlossen war. $\mathrm{Da}$ b von einer 'Vulgarisierung' von Bibelzitaten weder bei Irenaeus noch bei Novatian die Rede sein kann, verbürgt die mit Rëht gerühmte treffliche Überlieferung des lateinischen Irenaeus, beweist die grundsätzliche Übereinstimmung des erst jüngst wiederentdeckten ersten Novatiancodex mit dem Migne-Text hinsichtlich der Bibelzitate.

Mit den Namen des Irenaeus und Novatian nähern wir uns dem erstmöglichen Entstehungstermin von $d$ überhaupt: die gewonnenen Resultate gipfeln in den Thesen:

I) Der vg-Typus ist wesentlich älter als Hieronymus und lag schon um 250 vor.

2) Der $d$-Typus gehört spätestens der ersten Hälfte des $\Pi I J$ J.an.

3) Beide Textformen treten fast gleichzeitig in die Erscheinung.

1 Iren. $=\mathrm{d}: 5,3$ p. $308 \mathrm{Phil}$ 3, 21 s. S. 129. Iren. $=\mathrm{vg}: 3$, 20 I Cor I 29 in conspectac domini omnis caro : o. c. in consp. einus $\mathrm{vg}: 0$. c. coram deo d. 4, $15 \mathrm{I}$ Cor $i_{s}$ indulgentiam : consilium d. 4, 27 I Cor $10_{4}$ autem . . . consequenti eos : enim ... sequenti se d (so Iren. 4,14 außer eos). ebda $I$ Cor io 10 murmuraueritis . . . eorum : murmurenur ...ex illis d. 3, 24. 4, 26 I Cor $12{ }_{28}$ doctores : magistros d. 2, 28 I Cor 13 g quidem (enim vg) cognoscimius . . prophetamus : enim scimus . . prophetemur d (Iren. 4, 9 enim scimus . . prophetamus). 4, 27 I Cor 6 i1 et haec quidam (u. 1. quidem) fuistis : sed haec aliquando $f$. d. 5 , 14 Eph 2.15 euacuans : destituens d. I, 16 Tit $3_{10}$ post primam (unam vg) et secundam correptionem : post unam corr. et duo $\mathrm{d}$ (desgl. Iren. 3, 2).

${ }^{2}$ Nouatian. $=\mathrm{d}$ : de cib. iud. I Eph $6_{12}$ aduersus spiritalia : contra sp. vg. ebda 6 I Tim $6_{8}$ uictum et uestitum ... sumus : alimenta et quibus tegamur. . . . simus vg. Nouatian. = vg: de trin. $3 \mathrm{Rm} \mathrm{II}_{36}$ in ipso sunt omnia : in ipsum onnia d. ebda 29 I Cor 7 10 puto autem quia (quod vg) et ego : puto et ego autem d. "ebda 7 I Cor 2 , his qui diligunt illum : diligentibus eum d. ebda 29 I Cor $2{ }_{12}$ spiritum mundi... spiritum qui ex deo (eo Nouatian.) est : sp. huius mundi... spiritum dei d. de cib. iud. 5 Col $2_{21}$ ne tetigeritis $\langle. .$.$\rangle neque contrectaueritis : ne tangas... neque$ adtaminaueris $\mathrm{d}$. 
Wenn nun aber den vg-Typus ebenso wie die anderen Textzeugen vom Archetypus die verschollene 'Zwischenstufe' $d_{1}$ trennt, $\mathrm{muß} d_{1}$ in der ersten Hälfte des III Jh. entstanden sein, der $d$-Typus im II Jh., dieser zu Lebzeiten des Irenaeus, jener kurz nach des Bischofs Tode. Damals wurde der Lateiner frei (s. S. I I $f$.) und es entsproß aus $d_{1}$ als erster Ableger die $v g$-Form, deren älteste unverdächtige Kronzeugen Novatian und der Übersetzer des Irenaeus sind, wohl beide, ersterer ohne jeden $Z$ weifel, Zeitgenossen Tertullians und Cyprians.

$\mathrm{Daß}$ der lateinische Irenaeus zur Zeit der ersten Übersetzungsperiode ( $150-250$ ) entstanden, hat $\mathrm{H}$. Jordan bestritten. Unbestreitbar aber ist, daß der lateinische Paulustext des Irenaens latinus der Urform, wie sie aus $d$ vielfach zu uns spricht, näher steht als selbst Tertullian und Cyprian, der $d_{1}$ ohne Zweifel gekannt hat. Schon dieser Befund spricht für das höhere Alter des lateinischen Irenaeustextes. Nun stützt sich aber der Übersetzer da, wo Irenaeus ein Bibelzitat einschaltete, nachweislich auf einen lateinischen Bibeltext ohne Rücksicht auf das ihm vorliegende Original ${ }^{1}$. Dies Vorgehen mag uns und einer im Banne eines dogmatisch geheiligten Textes befangenen Zeit selbstverständlich erscheinen, Autoren des III Jh. und einer viel späteren Epöche lag es ebenso fern ${ }^{2}$. Es ist durchaus symptomatisch, wenn, von Hilarius, Ambrosius, Augustin u. v. a. zu schweigen, selbst Hieronymus die von ihm theoretisch so leidenschaftlich geforderte einheitliche Textgestalt der lateinischen Bibel in praxi in keiner Weise durchgeführt hat. $\mathrm{Er}$ war in dieser Beziehung nachsichtiger gegen sich selbst, als manch anderer christlicher Autor, z. B. Cyprian und Ambrosius.

Um so seltsamer berührt der Eigenwille des Urhebers des Irenaeus latinus, um so dringender verlangt man eine befriedigende Erklärung. Dogmatische Erwägungen, durch die sich selbst ein Hieronymus nicht fesseln.ließ, schalten aus. Je intensiver und länger

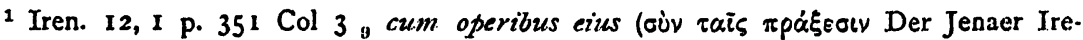
naeus-Papyrus hrsg. von H. Lietzmanu Nachr. d. GGdW I912 S. 316). Iren. 1, 1 I Tim

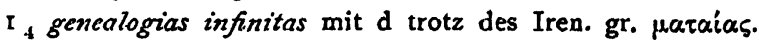

2 Die Arbeitsweise des uetzus interpres des Origenes (Rufin?) ist grundverschieden. Dieser bewabrte sich volle Bewegungsfreibeit gegenüber seincm Original, wie cs z. $B$. auch Rufin stets gehalten: er kürzte nach Belieben und machte Zusătze, die in Urtext nie standen: 2. B. in Mt 14,17 erweitert er Eph $5_{31}$ et crunt duo in carne una um den ganzen Vers 32. Sein Paulustext gebört zur d vg-bzw. d g-Klasse: in Mt 15, 3 I I Cor $2_{16}=\mathrm{d} \mathrm{vg} ; 15,31$ I Cor $2_{10}$ altitudines mit $\mathrm{g}$ (altitudinem $\mathrm{r}:$ alla $\mathrm{d}:$ pro. funda vg). 
man sich in das Werden des lateinischen Paulustextes vertieft, um so gebieterischer drängt sich die Überzeugung auf, daß den Irenaeus latinus trotz aller Abweichungen im einzelnen - ein Problem für sich - ein inneres Verhältnis mit der lateinischen Bibel verband. Ich stelle nebeneinander Irenaeus, $d$ und $v g$ zu Phil $\cdot 3{ }_{21}$ :

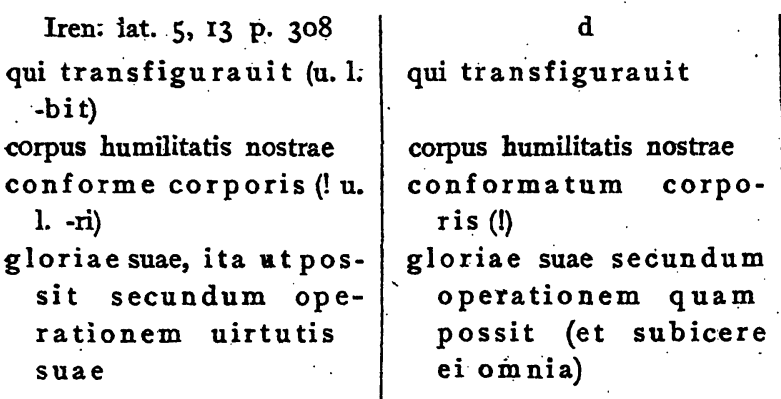

$\nabla \mathrm{g}$

qui reformabit $(\mu \in \tau \alpha \sigma \chi \eta$ -

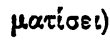

corpus humilitatis nostrae configuratum corpori

claritatis suae secundum operationem (t uirtutis suae vg S) qua etiam possit (possit etiam u. 1.) (subicere sibi omnia)

Der Tatbestand ist kurz folgender: Die absteigende Klimax Irenaeus $>d>v g$ ist unverkennbar. Beweis: conforme corporis : conformatum corporis (!) : configuratum corpori: die ursprüngliche

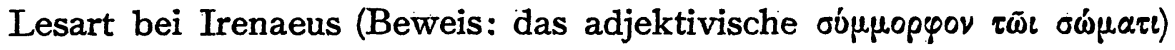
stand auch im Archetypus $d$ (Beweis: außer indirekten Zeugnissen, corporis!), das sekundäre Partizipium in $d$ wurde durch ein synonymes in der $v g$ ersetzt. Die bereits Cyprian bekannte Sonderlesart von $d$ steht in der lateinischen Übersetzung eines erläuternden Zusatzes des Irenaeus: quod igitur est humilitatis corpus, quod transfigurabit dominus conformatum corpori gloriae suae? Entlehnung einer Erklärung oder Umschreibung im Irenaeus. latinus aus diesem für den $d$-Text ohne triftigen Grund ist wenig glaublich. Sie ist gerechtfertigt und begreiflich, wenn der Übersetzer des Irenaeus zum $d$-Text in naher Beziehung stand. Dann leuchtet es auch ein, weshalb er auf Kosten seiner griechischen Vorlage einen bestimmten lateinischen Paulus- und Bibeltext bevorzugte. War es etwa der Übersetzer selbst oder einer seines Kreises, der die an das Apostolos-Exemplar des Lyoneser Bischofs sich anlehnende lateinische Urübersetzung frei machte und revidierte, also $d_{1}$ schuf? Und sollte dieser Redaktor das voraufgehende Evangeliar völlig unberührt gelassen haben? Der Übersetzer des Irenaeus sah aber auch einen $v g$-Text ein, der als erster Nachkomme von $d_{1}$ bald nach $\mathrm{d}_{1}$ entstanden war.

Für die vg trat damals als vornehmster Zeuge Nouatianus auf, Roms erster lateinischer Kirchenschriftsteller, der

Zeitschr. f. d. nentest. Wiss. 20. Band $192 x$. 
Freund Cyprians, dem er vielleicht ein Exemplar des ersten 'lateinischen' NT (Evang. und Paulus) verehrte. Denn $d_{1}$ war für ihn ebensowenig eine unbekannte Größe wie $d$ und das Werk des Irenaeus gegen die Gnosis. - Der lateinischen Bearbeitung dieses zum Schutze der Rechtgläubigkeit verfaßten Werkes mochte Novatian, der eifrige Hüter des Wortes der Schrift, lebhaftes Interesse entgegengebracht haben. Das aktuellste Problem jener Zeit aber war, die hl. Schriften der jungen Religion den Römern in ihrer Muttersprache, und zwar in möglichst' lesbarer Gestalt, zugänglich zu machen.

$\mathrm{Daß}$ dieses Problem gerade zu Novatians Zeiten dank der vg für den lateinischen Paulus gelöst wurde, ist erwiesen. Daß damals noch der Kreis derer, die willens und befähigt waren, die literarischen Schätze des frühen Christentums in ein gefälliges lateinisches Gewand zu kleiden, überaus klein war, ist nicht zu bezweifeln. $\mathrm{DaB}$ derartige Versuche sich auf die Paulinischen Briefe beschränkt, die Evangelien aber ignoriert hätten, ist ausgeschlossen. Daß Nouatianus, der literarisch feingebildete Mann und die bedeutendste Persönlichkeit im römischen Klerus jener Tage, diesen literarischen Bestrebungen fern stand, ist höchst unwahrscheinlich. Das Gegenteil dürfte der Wahrheit näher kommen. Das erst in der ersten Hälfte des III Jh. zum nationalen Selbstbewubtsein. erwachende christliche Rom - es ist gewiß kein Zufall, wenn die Grabschrift des Papstes Cornelius als erste und einzigste im III Jh. in lateinischer Sprache und mit lateinischen Lettern geschrieben wurde mochte in Novatian den eifrigen Förderer und geistigen Urheber 'seines' Bibeltextes begrüßt haben, jenes Textes, dem dạnn Hieronymus die endgültige Gestalt gab.

Jetzt erst verstehen wir, weshalb bei der Nachwelt der Name dessen, der dem lateinischen Paulus und wohl auch den lateinischen Evangelien eine fast $z$ wei Jahrtausende überdauernde persönliche Note aufprägen. ließ, der damnata memoria verfiel, weshalb Hieronymus über Ursprung und Eigenart des seiner Recensio zugrunde gelegten lateinischen Evangeliars sich gänzlich ausschweigt: Rom und er hätten bekennen müssen, dal der in Rom übliche und gültige Text der Hauptbücher des NT letzten Endes das Werk und das Verdienst eines Schismatikers waren. Wir verstehen aber auch, weshalb in der Folgezeit bei der Vormachtstellung der romischen Kirche niemand am 'römischen' Text (Augustins itala?l) vorübergehen konnte.

Den tatsächlichen und geistigen Urhebern der vg und des 
Irenaeus latinus - letzterer hatte es ja auch abgelehnt, seinen lateinischen Bibeltext durch die griechischen Zitate im é $\lambda \varepsilon \gamma \chi 0 s$ bevormunden zu lassen - erwuchs aus ibren Taten das Recht, sowohl $d$ als $v g$ nach Belieben zu zitieren, selbst einen Kompromißtext aus $d$ und $v g^{1}$.

Den lateinischen Text der Paulinischen Briefe überhaupt dankt die Welt einem oder dem 'Apostolos'-Exemplar des großen christlichen Apologeten von Lyon, der ersten Vorlage des Cläromontanus, den, mit dem Evangeliar vereint, als kostbarstes Kleinod einstmals das Kloster des Irenaeus zu Lyon barg. Aber nicht nur das griechische und lateinische 'Urbild' des lateinischen Paulus 'hat uns Irenaeus beschieden, auf ihn und seinen Namen weisen auch die gründlichsten und folgenschwersten Revisionen des $d$-Typus zu $d_{1}$, von $d_{1}$ zur Urform der vg: sie weisen in die Zeit und den Kreis der Männer, welche der Nachwelt das für den Westen fremdsprachige Wèrk des streitbaren Bischofs gerettet haben, den Irenaeıs latinus.

\section{Nachtrag.}

Harnacks 'Studien zur Vulgata des Hebrä̈erbriefs' kamen infolge eines leidigen Mibverständnisses auf Grund der Nachricht von der Not der deutschen Wissenschaft und der in der pr. Staatsbibliothek niedergelegten Arbeit erst nach Abschluß des MS. und dessen Versendung an den Hrsg. in meine Hände. Erfreulicherweise hält auch $H$. v. Harnack die Abhängigkeit der vg von $d$ für erwiesen, auch für ihn ist die $v g$ eine Repräsentantin der 'Itala': die Basis, auf der sich die vorstehende Untersuchung aufbaut (S. $102 \mathrm{f}$. u. II I). Alsdann abèr spricht $H$. der Überlieferung des lateinischen Hebr. eine Sonderstellung zu gegenüber den 'Paulinischen' Briefen oder hält sie doch für möglich, und läßt vor allem bei der Recensio des Vulgatatextes Hieronymus neben stilistischen Korrekturen den $r$-Text befragen.

Leider durfte hier infolge Raummangels aus reichbaltigstem bzw. vollständigem Material, dessen Bewertung erst nach durchgeführter Recensio möglich wurde, nur das unumgänglich Notwendige vorgelegt werden, mehrfach war sogar gänzliche Unterdrückung von Belegen geboten: so S. 112 für die allen Texten im Gegensatz zu $d$ gemeinsamen synonymen Begriffe und Ausdrucksformen, S. I15 unten für die allenthalben gleichlautend verbesserten

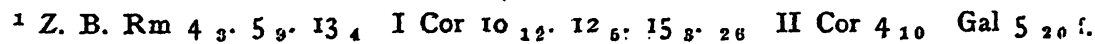
Eph 5 . Col $3_{5}$ II Thess 2 . 
Fehler von $d$, S. $118 \mathrm{f}$. 12 I für die Gruppen $g r ; g v g, g$ Ambrst., $r v g, r$ Ambrst. u. s. f., S. I20. I2 I für die Sonderlesarten jedes einzelnen Textzeugen u. a. $\mathrm{m}$.

Auch nach Kenntnisnahme der Untersuchungen Harnacks wir fußen selbstverständlich auf demselben Quellenmaterial - halte ich auch für die Überlieferungsgeschichte des lat. Hebr, auf dessen Eigenart ich S. $99 \mathrm{f}$. hinwies, die folgenden Thesen aufrecht:

I) Eine Sonderstellung kommt dem lat. Hebr, der vom Claromontanus $D d$ unzertrennlich ist, in seinem Werdegang im Gegensatz $\mathrm{zu}$ den übrigen Paulusbriefen nicht $z u$.

2) Der Urtypus des $d$-Textes des Hebr war, wie $d$ noch heute beweist, infolge der Schwierigkeiten des griechischen Originals im Vergleich zu den übrigen Paulinischen. Briefen in erhöhtem Maße schwerfällig, unbeholfen, fehlerhaft. Die notwendige Folge war die ungemein große Verschiedenheit von $d: v g, d: r, v g: r$.

3) Auch die $v g$-Form des Hebr war, ehe der $r$-Typus geprägt wurde, also auch älter als Hieronymus.

4) Sowohl die $r$-Form, als die $v g$-Form des Hebr sind aus dem $d$-Typus (bzw. $d_{1}$ ) - und zwar jeder für sich - hervorgegangen. $r$ ist gegenüber $d$ nicht eignen Rechts.

5) Auch der $r$-Text des Hebr ist ohne die $v g$-Form nicht $z u$ verstehen. Sein Urheber hat den lateinischen Text bearbeitet, ihn aber keineswegs nach einer griechischen Vorlage syste$m$ atisch redigiert, geschweige aus einem griechischen Exemplar selbständig übersetzt.

6) Für die Mehrzahl der $r$-Lesarten ist der lateinische Redaktor verantwortlich. Erst in zweiter Linie wurde ein griechischer Paulus befragt, wenn es galt, wirkliche oder vermeintliche Unebenheiten des lateinischen Textes auszumerzen.

7) Einen Cod. $R$ im Gegensatz zu $D E(G F W)$ - laurer $D$ Texten - hat es auch für Hebr nie gegeben.

[Abgeschlossen am 1. März 19'21.] 